CRYSTALLOGRAPHIC COMMUNICATIONS

ISSN 2056-9890

Received 12 November 2018

Accepted 26 November 2018

Edited by J. Simpson, University of Otago, New Zealand

Keywords: crystal structure; hydrates; hydrogen bonding; tautomerism.

CCDC references: $1881120 ; 1881119$

Supporting information: this article has supporting information at journals.iucr.org/e

\section{Structures of the hydrate and dihydrate forms of the DNA-binding radioprotector methylproamine}

\author{
Jonathan Michael White,* Samuel Charles Brydon and Thomas Fellowes \\ School of Chemistry and BIO-21 Institute, University of Melbourne, Parkville, VIC 3010, Melbourne, Australia. \\ *Correspondence e-mail: whitejm@unimelb.edu.au
}

Methyl proamine $\left\{N, N, 3\right.$-trimethyl-4-[6-(4-methylpiperazin-1-yl)- $1 H, 3^{\prime} H$-[2,5' bibenzo[d]imidazol]-2'-yl]aniline, $\mathrm{C}_{28} \mathrm{H}_{35} \mathrm{~N}_{7} \mathrm{O}_{2}$, crystallized as both a dihydrate, $\mathrm{C}_{28} \mathrm{H}_{31} \mathrm{~N}_{7} \cdot 2 \mathrm{H}_{2} \mathrm{O}$, and monohydrate, $\mathrm{C}_{28} \mathrm{H}_{31} \mathrm{~N}_{7} \cdot \mathrm{H}_{2} \mathrm{O}$, form from water in the presence of $\beta$-cyclodextrin, in the $P 2_{1} / c$ and $P 2_{1} / n$ space groups, respectively. The two structures adopt different conformations and tautomeric forms as a result of the differing crystal packing as dictated by hydrogen-bonding interactions. The dihydrate crystallizes as a three-dimensional hydrogen-bonded network, while the monohydrate crystallizes as a two-dimensional hydrogenbonded network.

\section{Chemical context}

Methylproamine (1) is a bibenzimidazole derivative which binds in the minor groove of DNA in adenine-thymine-rich regions of four or more consecutive AT pairs (Martin et al., 2004) and is related to the Hoechst family of DNA-binding bibenzimidazoles (Pjura et al., 1987). Although the structure of methylproamine with the DNA dodecamer $\mathrm{d}$ (CGCGAATTCGCG $)_{2}$ has been determined and reported by us, the structure of the free ligand has not yet been published as it is very difficult to obtain good quality crystals for these types of compounds. In order to examine the conformational and tautomeric differences between the uncomplexed ligand and that which is bound to DNA, the structures of both the dihydrate (1) $\cdot 2 \mathrm{H}_{2} \mathrm{O}$ and the monohydrate (1) $\mathrm{H}_{2} \mathrm{O}$, which were grown from water in the presence of $\beta$-cyclodextrin, are reported.

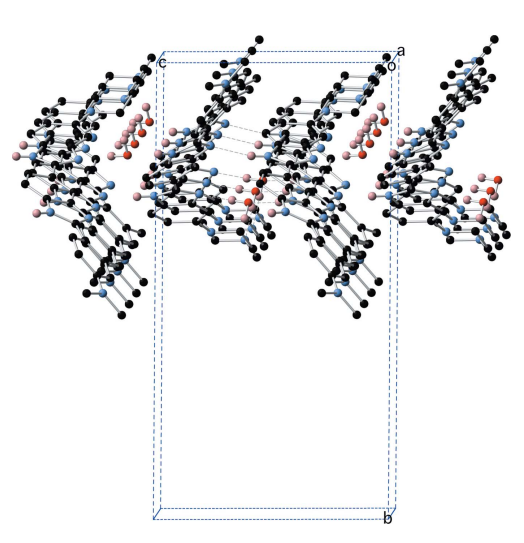<smiles></smiles>

(1) - Dihydrate<smiles>Cc1cc(N(C)C)ccc1-c1nc2ccc(-c3nc4ccc(N5CCN(C)CC5)cc4[nH]3)cc2[nH]1</smiles> 
Table 1

Selected geometric parameters $\left(\AA{ }^{\circ}\right)$ for $(1) \cdot 2 \mathrm{H}_{2} \mathrm{O}$.

\begin{tabular}{lclc}
\hline $\mathrm{C} 1-\mathrm{C} 7$ & $1.4698(18)$ & $\mathrm{C} 10-\mathrm{C} 14$ & $1.4701(19)$ \\
$\mathrm{C} 4-\mathrm{N} 1$ & $1.3923(18)$ & $\mathrm{C} 14-\mathrm{N} 4$ & $1.3708(18)$ \\
$\mathrm{C} 7-\mathrm{N} 2$ & $1.3695(17)$ & $\mathrm{C} 14-\mathrm{N} 5$ & $1.3215(18)$ \\
$\mathrm{C} 7-\mathrm{N} 3$ & $1.3325(18)$ & $\mathrm{C} 17-\mathrm{N} 6$ & $1.4321(19)$ \\
& & & \\
$\mathrm{C} 4-\mathrm{N} 1-\mathrm{C} 27$ & $118.18(13)$ & $\mathrm{C} 17-\mathrm{N} 6-\mathrm{C} 24$ & $114.80(13)$ \\
$\mathrm{C} 4-\mathrm{N} 1-\mathrm{C} 26$ & $118.60(13)$ & $\mathrm{C} 17-\mathrm{N} 6-\mathrm{C} 21$ & $113.32(12)$ \\
$\mathrm{C} 27-\mathrm{N} 1-\mathrm{C} 26$ & $112.78(13)$ & $\mathrm{C} 24-\mathrm{N} 6-\mathrm{C} 21$ & $109.48(12)$ \\
& & & \\
$\mathrm{C} 2-\mathrm{C} 1-\mathrm{C} 7-\mathrm{N} 3$ & $-30.0(2)$ & $\mathrm{C} 9-\mathrm{C} 10-\mathrm{C} 14-\mathrm{N} 4$ & $173.54(14)$ \\
$\mathrm{C} 6-\mathrm{C} 1-\mathrm{C} 7-\mathrm{N} 3$ & $147.26(15)$ & $\mathrm{C} 3-\mathrm{C} 4-\mathrm{N} 1-\mathrm{C} 27$ & $1.2(2)$ \\
$\mathrm{C} 2-\mathrm{C} 1-\mathrm{C} 7-\mathrm{N} 2$ & $155.44(14)$ & $\mathrm{C} 5-\mathrm{C} 4-\mathrm{N} 1-\mathrm{C} 26$ & $40.9(2)$ \\
$\mathrm{C} 6-\mathrm{C} 1-\mathrm{C} 7-\mathrm{N} 2$ & $-27.3(2)$ & $\mathrm{C} 16-\mathrm{C} 17-\mathrm{N} 6-\mathrm{C} 24$ & $-170.61(14)$ \\
$\mathrm{C} 9-\mathrm{C} 10-\mathrm{C} 14-\mathrm{N} 5$ & $-5.7(2)$ & $\mathrm{C} 16-\mathrm{C} 17-\mathrm{N} 6-\mathrm{C} 21$ & $62.58(19)$ \\
\hline
\end{tabular}

\section{Structural commentary}

Displacement ellipsoid plots for (1) $2 \mathrm{H}_{2} \mathrm{O}$ and (1) $\cdot \mathrm{H}_{2} \mathrm{O}$ are presented in Figs. 1 and 2, respectively. The two structures represent two different conformations of (1); (1) $2 \mathrm{H}_{2} \mathrm{O}$ exists in an extended conformation as determined by the $\mathrm{C} 9-\mathrm{C} 10-$ $\mathrm{C} 14-\mathrm{N} 4$ torsion angle which is $173.54(14)^{\circ}$ with an $\mathrm{N} 1 \cdots \mathrm{N} 6$ distance of 17.251 (2) Å while (1) $\cdot \mathrm{H}_{2} \mathrm{O}$ adopts a crescent shape with a $\mathrm{C} 9-\mathrm{C} 10-\mathrm{C} 14-\mathrm{N} 4$ torsion angle of $-19.8(2)^{\circ}$ and an $\mathrm{N} 1 \cdots \mathrm{N} 6$ distance of 16.859 (2) $\AA$. In addition, they represent different tautomeric forms of (1); (1) $\cdot 2 \mathrm{H}_{2} \mathrm{O}$ can be described as the $\mathrm{N} 2, \mathrm{~N} 4$ tautomer whereas (1) $\cdot \mathrm{H}_{2} \mathrm{O}$ exists in the crystal as the $\mathrm{N} 2, \mathrm{~N} 5$ tautomer as defined by the numbering scheme used in Figs. 1 and 2. The tautomeric form adopted in each case is implied not only by the $\mathrm{N}-\mathrm{H}$ hydrogen atoms, which were located in difference maps and refined satisfactorily without restraint, but also by the $\mathrm{C}-\mathrm{N}$ bond distances of the two benzimidazole moieties within the structures (Tables 1 and 2). The tautomeric form assigned in each case is also supported by the intermolecular hydrogen bonds that these $\mathrm{N}-\mathrm{H}$ groups participate in. It is the intermolecular hydrogen-

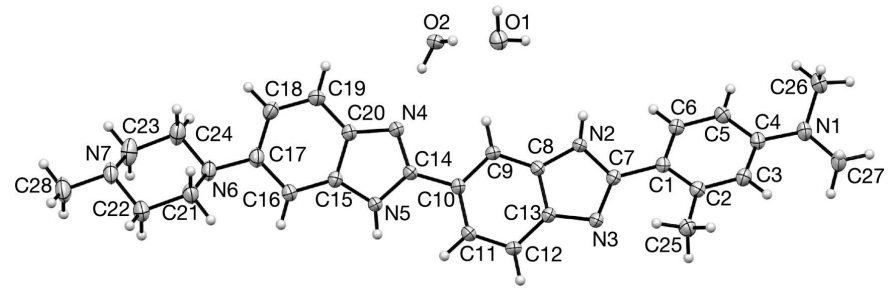

Figure 1

Displacement ellipsoid plot of the asymmetric unit for dihydrate (1) $\cdot 2 \mathrm{H}_{2} \mathrm{O}$.

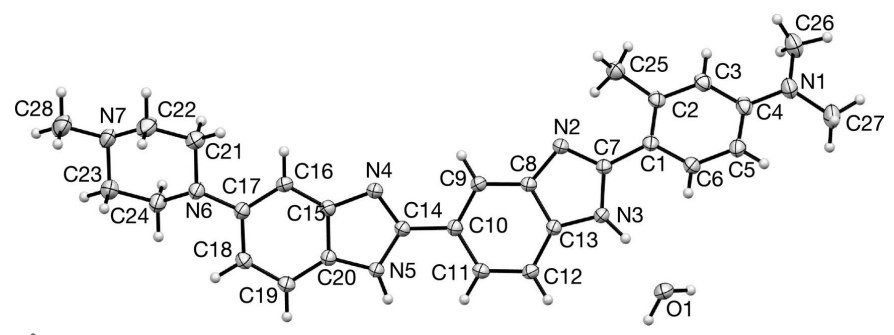

Figure 2

Displacement ellipsoid plot of the asymmetric unit for hydrate (1) $\mathrm{H}_{2} \mathrm{O}$.
Table 2

Selected geometric parameters $\left(\AA,^{\circ}\right)$ for $(1) \cdot \mathrm{H}_{2} \mathrm{O}$.

\begin{tabular}{lclc}
\hline $\mathrm{C} 1-\mathrm{C} 7$ & $1.468(2)$ & $\mathrm{C} 10-\mathrm{C} 14$ & $1.464(2)$ \\
$\mathrm{C} 4-\mathrm{N} 1$ & $1.374(2)$ & $\mathrm{C} 14-\mathrm{N} 4$ & $1.3286(19)$ \\
$\mathrm{C} 7-\mathrm{N} 2$ & $1.3344(19)$ & $\mathrm{C} 14-\mathrm{N} 5$ & $1.3691(19)$ \\
$\mathrm{C} 7-\mathrm{N} 3$ & $1.3687(19)$ & $\mathrm{C} 17-\mathrm{N} 6$ & $1.4190(19)$ \\
& & & \\
$\mathrm{C} 4-\mathrm{N} 1-\mathrm{C} 27$ & $120.55(15)$ & $\mathrm{C} 17-\mathrm{N} 6-\mathrm{C} 21$ & $117.43(13)$ \\
$\mathrm{C} 4-\mathrm{N} 1-\mathrm{C} 26$ & $119.35(15)$ & $\mathrm{C} 17-\mathrm{N} 6-\mathrm{C} 24$ & $115.25(13)$ \\
$\mathrm{C} 27-\mathrm{N} 1-\mathrm{C} 26$ & $119.08(14)$ & $\mathrm{C} 21-\mathrm{N} 6-\mathrm{C} 24$ & $109.98(13)$ \\
$\mathrm{C} 2-\mathrm{C} 1-\mathrm{C} 7-\mathrm{N} 2$ & $-23.6(2)$ & $\mathrm{C} 9-\mathrm{C} 10-\mathrm{C} 14-\mathrm{N} 5$ & $161.50(14)$ \\
$\mathrm{C} 2-\mathrm{C} 1-\mathrm{C} 7-\mathrm{N} 3$ & $154.23(14)$ & $\mathrm{C} 16-\mathrm{C} 17-\mathrm{N} 6-\mathrm{C} 21$ & $3.4(2)$ \\
$\mathrm{C} 9-\mathrm{C} 10-\mathrm{C} 14-\mathrm{N} 4$ & $-19.8(2)$ & $\mathrm{C} 18-\mathrm{C} 17-\mathrm{N} 6-\mathrm{C} 24$ & $-48.47(19)$ \\
\hline
\end{tabular}

bonded interactions involving these $\mathrm{N}-\mathrm{H}$ groups which no doubt play a major role in which tautomer is adopted in each case in the solid state.

In both structures the ortho-methyl substituent in ring $A$ lies on the opposite side of the structure to the $\mathrm{N}-\mathrm{H}$ hydrogen atom of benzimidazole ring $B$, this is very likely for steric reasons; the dihedral angles between the two rings as defined by $\mathrm{C} 2-\mathrm{C} 1-\mathrm{C} 7-\mathrm{N} 3$ in (1) $2 \mathrm{H}_{2} \mathrm{O}$ and by $\mathrm{C} 2-\mathrm{C} 1-\mathrm{C} 7-\mathrm{N} 2$ in (1) $\cdot \mathrm{H}_{2} \mathrm{O}$, which are $-30.0(2)$ and $-23.6(2)^{\circ}$, respectively, reflect a balance between electronic effects which prefer coplanarity between the two aromatic rings and steric effects whereby the ortho-methyl group would be unreasonably close to the benzimidazole nitrogen of ring $B$. The dihedral angles between the two benzimidazole rings (rings $B$ and $C$ ) are $-5.7(2)$ and $-19.8(2)^{\circ}$, respectively.

The geometry of the para-dimethylamino substituent on ring $A$ differs between the two structures; the mean $\mathrm{C}-\mathrm{N} 1-\mathrm{C}$ angles are 116.4 and $119.7^{\circ}$, respectively, for (1) $2 \mathrm{H}_{2} \mathrm{O}$ and (1) $\cdot \mathrm{H}_{2} \mathrm{O}$, suggesting that the former is more pyramidalized, consistent with this are the significant differences in the $\mathrm{C} 4-$ $\mathrm{N} 1$ bond distances which are 1.3923 (18) and 1.374 (2) $\AA$ for (1) $\cdot 2 \mathrm{H}_{2} \mathrm{O}$ and (1) $\cdot \mathrm{H}_{2} \mathrm{O}$, respectively.

It is interesting to compare the conformation of (1) in these two structures with that adopted by (1) when bound in the minor groove of the palindromic DNA dodecamer [d(CGCGAATTCGCG) $)_{2}$; Martin et al., 2004]. The ligand must adopt the 2-H, 4-H tautomeric form with a crescent shape similar to that adopted by (1) $\cdot \mathrm{H}_{2} \mathrm{O}$ so that it can direct the necessary $\mathrm{N}-\mathrm{H}$ hydrogen-bond donors into the minor groove, in addition the ortho-methyl substituent on ring $A$ must be facing away from the crescent. A superposition of the two structures with that of (1) bound to DNA is shown in Fig. 3.

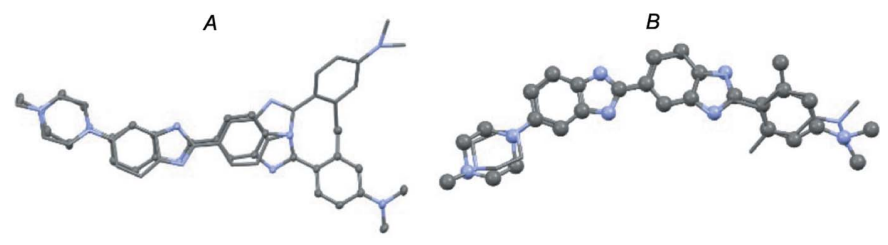

Figure 3

Overlay for the structures of $A$; (1) $\cdot 2 \mathrm{H}_{2} \mathrm{O}$ and $B ;(1) \cdot \mathrm{H}_{2} \mathrm{O}$ with DNAbound (1). In the LH-structure the DNA-bond ligand is indicated by capped sticks, while in the RH structure it is ball and stick. 
Table 3

Hydrogen-bond geometry $\left(\AA,^{\circ}\right)$ for $(1) \cdot 2 \mathrm{H}_{2} \mathrm{O}$.

\begin{tabular}{|c|c|c|c|c|}
\hline$D-\mathrm{H} \cdots A$ & $D-\mathrm{H}$ & $\mathrm{H} \cdots A$ & $D \cdots A$ & $D-\mathrm{H} \cdots A$ \\
\hline $\mathrm{C} 23-\mathrm{H} 23 B \cdots \mathrm{N} 2^{\mathrm{i}}$ & 0.99 & 2.65 & $3.542(2)$ & 149 \\
\hline $\mathrm{O} 1-\mathrm{H} 1 A \cdots \mathrm{O} 2^{\mathrm{ii}}$ & $0.91(3)$ & $1.98(3)$ & $2.8665(17)$ & $164(2)$ \\
\hline $\mathrm{O} 1-\mathrm{H} 1 B \cdots \mathrm{N} 7^{\mathrm{iii}}$ & $0.94(3)$ & $1.91(3)$ & $2.8482(18)$ & $170(3)$ \\
\hline $\mathrm{N} 2-\mathrm{H} 2 \cdots \mathrm{O} 2^{\mathrm{ii}}$ & 0.856 (18) & $1.944(18)$ & 2.7797 (15) & $165.0(17)$ \\
\hline $\mathrm{O} 2-\mathrm{H} 2 A \cdots \mathrm{N} 5$ & $0.86(2)$ & $1.91(2)$ & 2.7685 (16) & $175(2)$ \\
\hline $\mathrm{O} 2-\mathrm{H} 2 B \cdots \mathrm{O} 1$ & $0.90(3)$ & $1.86(3)$ & 2.7537 (18) & $168(3)$ \\
\hline $\mathrm{N} 4-\mathrm{H} 4 A \cdots \mathrm{N} 3^{\mathrm{iv}}$ & 0.870 (19) & $2.072(19)$ & $2.9411(16)$ & 176.7 (17) \\
\hline
\end{tabular}

Table 4

Hydrogen-bond geometry $\left(\AA,^{\circ}\right)$ for $(1) \cdot \mathrm{H}_{2} \mathrm{O}$.

\begin{tabular}{lllll}
\hline$D-\mathrm{H} \cdots A$ & $D-\mathrm{H}$ & $\mathrm{H} \cdots A$ & $D \cdots A$ & $D-\mathrm{H} \cdots A$ \\
\hline $\mathrm{O} 1-\mathrm{H} 1 A \cdots \mathrm{N} 7^{\mathrm{i}}$ & $0.93(4)$ & $1.93(4)$ & $2.858(2)$ & $177(3)$ \\
$\mathrm{O} 1-\mathrm{H} 1 B \cdots \mathrm{N} 4^{\mathrm{ii}}$ & $0.95(3)$ & $1.94(3)$ & $2.8905(18)$ & $177(2)$ \\
$\mathrm{N} 3-\mathrm{H} 3 A \cdots \mathrm{O} 1$ & $0.93(2)$ & $1.82(2)$ & $2.7338(17)$ & $170(2)$ \\
$\mathrm{N} 5-\mathrm{H} 5 A \cdots \mathrm{N} 2^{\mathrm{iii}}$ & $0.89(2)$ & $2.15(2)$ & $3.0199(18)$ & $167.2(18)$ \\
$\mathrm{O} 1-\mathrm{H} 1 A \cdots \mathrm{N} 7^{\mathrm{i}}$ & $0.93(4)$ & $1.93(4)$ & $2.858(2)$ & $177(3)$ \\
$\mathrm{O} 1-\mathrm{H} 1 B \cdots \mathrm{N} 4^{\mathrm{ii}}$ & $0.95(3)$ & $1.94(3)$ & $2.8905(18)$ & $177(2)$ \\
$\mathrm{N} 3-\mathrm{H} 3 A \cdots \mathrm{O} 1$ & $0.93(2)$ & $1.82(2)$ & $2.7338(17)$ & $170(2)$ \\
$\mathrm{N} 5-\mathrm{H} 5 A \cdots \mathrm{N} 2^{i \mathrm{ii}}$ & $0.89(2)$ & $2.15(2)$ & $3.0199(18)$ & $167.2(18)$ \\
\hline
\end{tabular}

Symmetry codes: (i) $\quad x-\frac{3}{2},-y+\frac{1}{2}, z+\frac{1}{2} ; \quad$ (ii) $\quad x-\frac{1}{2},-y+\frac{1}{2}, z+\frac{1}{2}$; $\quad$ (iii) $x+\frac{1}{2},-y+\frac{1}{2}, z+\frac{1}{2}$.

\section{Supramolecular features}

The structure of the dihydrate $(1) \cdot 2 \mathrm{H}_{2} \mathrm{O}$ is characterized by the presence of a centrosymmetric water tetramer which provides a template around which the structure is built. This

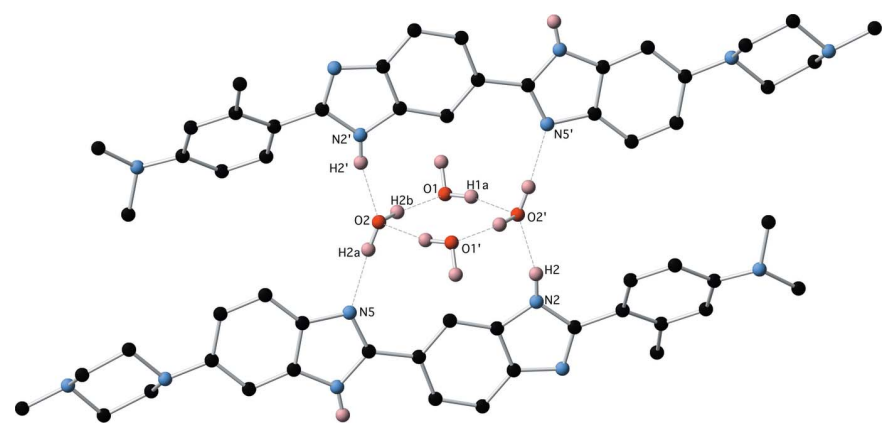

Figure 4

The water tetramer with diagonally hydrogen-bonded molecules of (1).

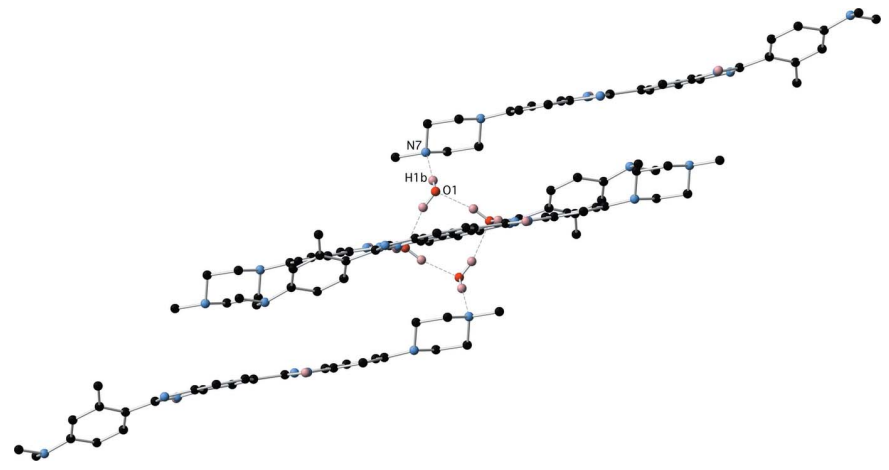

Figure 5

The water tetramer with additional hydrogen-bonded interactions with (1).

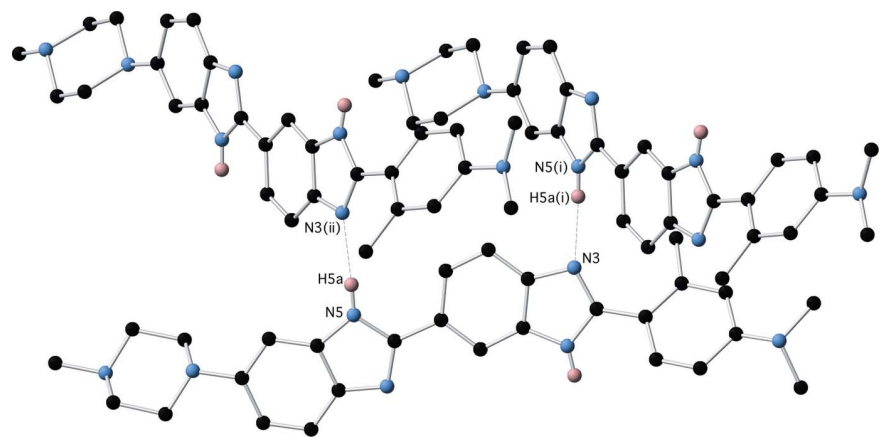

Figure 6

The cross-linking $\mathrm{N}-\mathrm{H} \cdots \mathrm{N}$ hydrogen bonds between benzimidazole moieties molecules of (1).

tetramer appears to be a common motif formed in crystalline hydrates with over 3689 examples of structures containing this motif in the Cambridge Structural Database [Version 1.23 update 5.39 (August 2018); Groom et al., 2016]. The water tetramer is bridged across opposite diagonals by two molecules of (1) by a combination of $\mathrm{N}-\mathrm{H} \cdots \mathrm{O}$ and $\mathrm{O}-\mathrm{H} \cdots \mathrm{N}$ hydrogen bonds involving the two benzimidazole groups (Fig. 4 and Table 3), the remaining $\mathrm{O}-\mathrm{H}$ hydrogens form $\mathrm{O}-$ $\mathrm{H} \cdot \mathrm{N}$ hydrogen bonds to two further centrosymmetrically related molecules of (1) via the tertiary piperazine nitrogen N7 (Fig. 5 and Table 3). This cluster of four molecules of (1) and the water tetramer is then extensively cross-linked by $\mathrm{N}-$

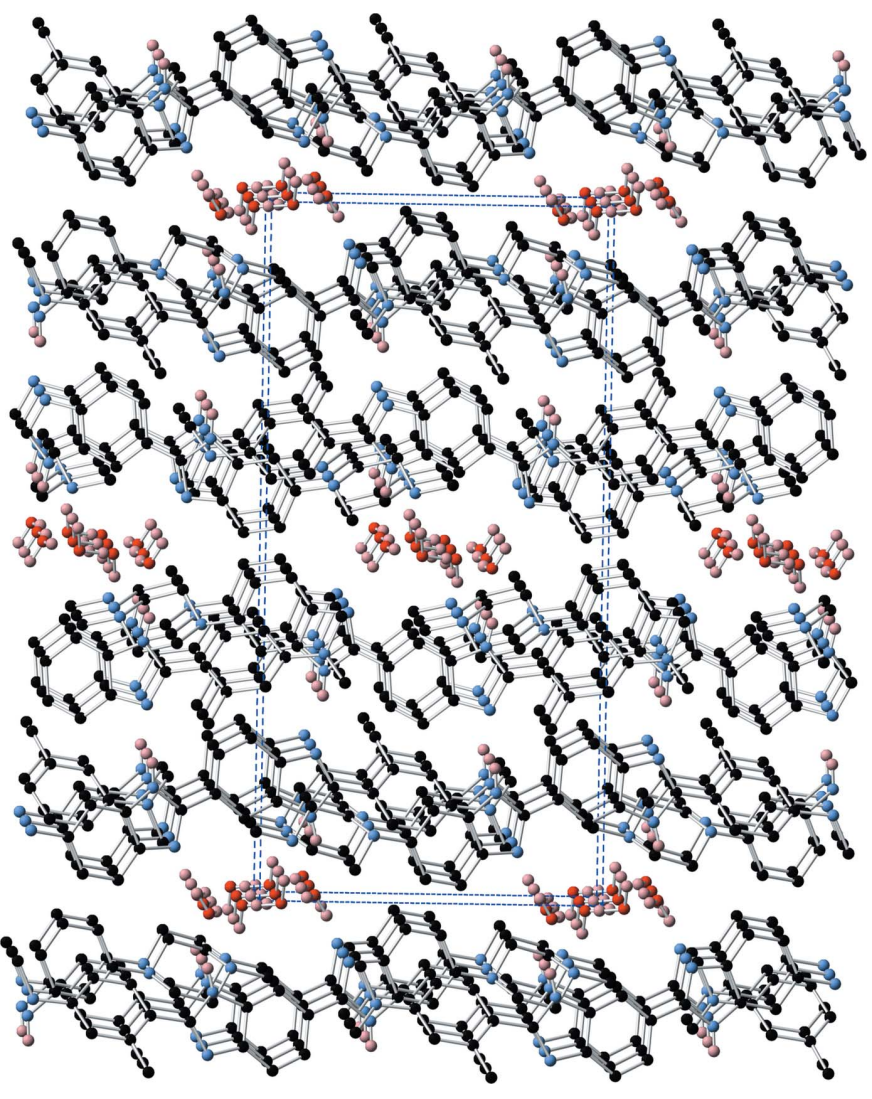

Figure 7

Three-dimensional hydrogen-bonded network in (1) $2 \mathrm{H}_{2} \mathrm{O}$. 


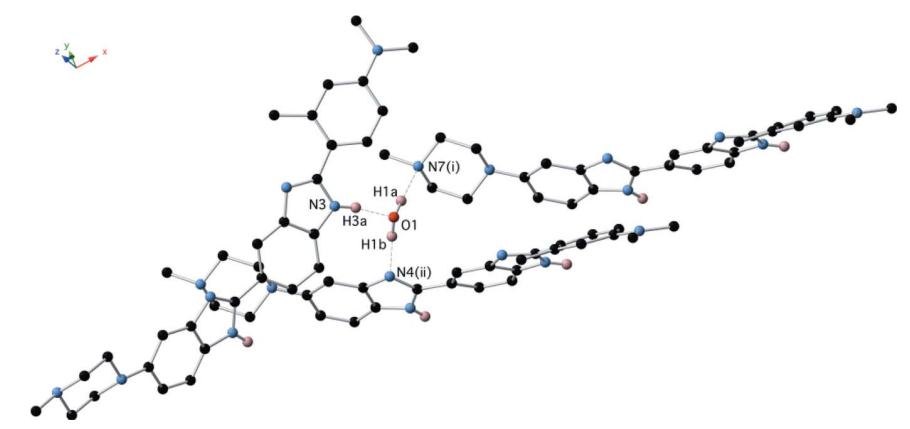

Figure 8

Hydrogen bonding between (1) and the water molecule in (1) $\cdot \mathrm{H}_{2} \mathrm{O}$.

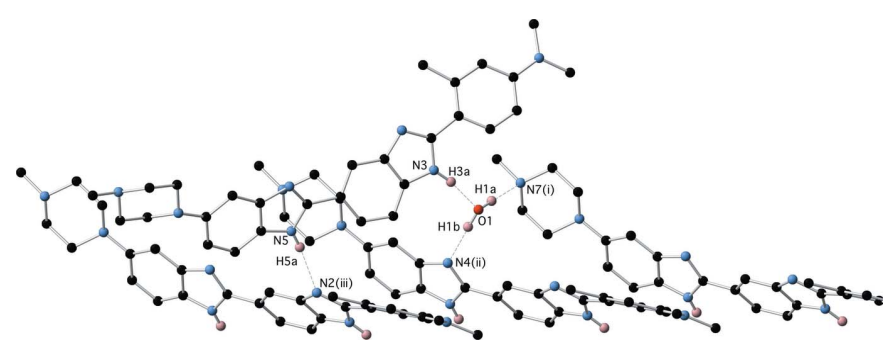

Figure 9

Direct $\mathrm{N}-\mathrm{H} \cdots \mathrm{N}$ hydrogen bonds between benzimidazole moieties of (1) $\cdot \mathrm{H}_{2} \mathrm{O}$.

$\mathrm{H} \cdot \mathrm{N}$ hydrogen bonds between the remaining benzimidazole groups (Figs. 6 and 7 and Table 3).

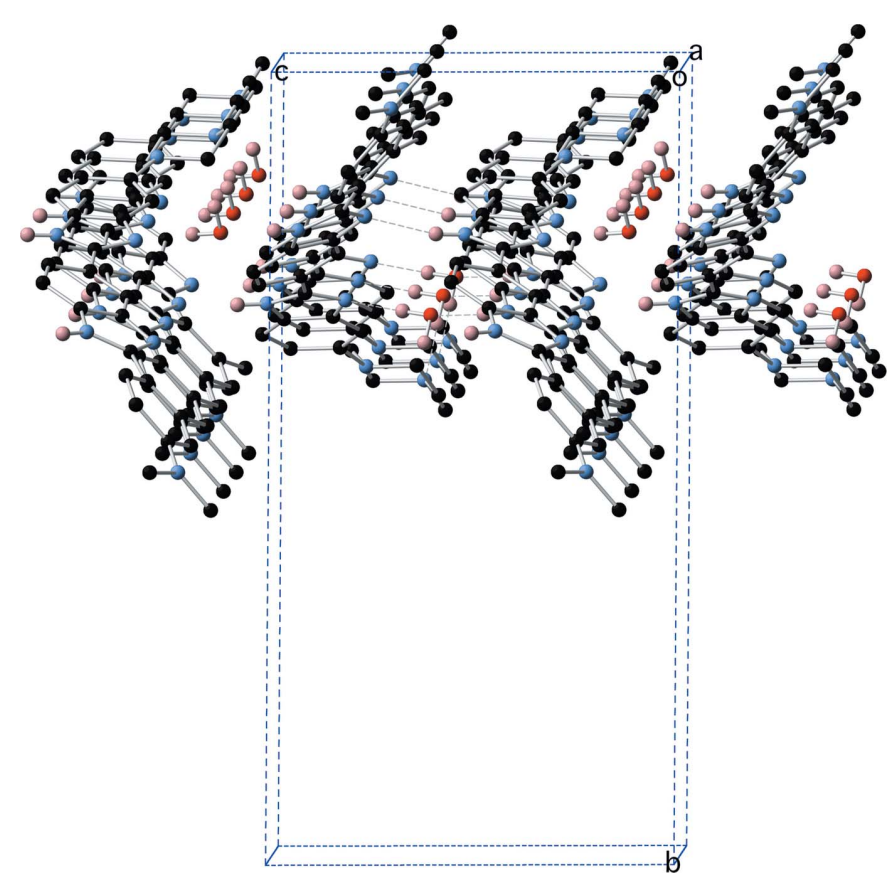

Figure 10

Two-dimensional hydrogen-bonded network in (1) $\cdot \mathrm{H}_{2} \mathrm{O}$.

The structure of the hydrate $(1) \cdot \mathrm{H}_{2} \mathrm{O}$ is also characterized by extensive hydrogen-bonding interactions, both directly between the benzimidazole moieties of (1), and via the water

Table 5

Experimental details.

(1) $2 \mathrm{H}_{2} \mathrm{O}$

Crystal data

Chemical formula

$M_{\mathrm{r}}$

Crystal system, space group

Temperature (K)

$a, b, c(\AA)$

$\beta\left({ }^{\circ}\right)$

$V\left(\AA^{3}\right)$

$Z$

Radiation type

$\mu\left(\mathrm{mm}^{-1}\right)$

Crystal size (mm)

Data collection

Diffractometer

Absorption correction

$T_{\min }, T_{\max }$

No. of measured, independent and observed $[I>2 \sigma(I)]$ reflections

$R_{\text {int }}$

$(\sin \theta / \lambda)_{\max }\left(\AA^{-1}\right)$
$\mathrm{C}_{28} \mathrm{H}_{31} \mathrm{~N}_{7} \cdot 2 \mathrm{H}_{2} \mathrm{O}$

501.63

Monoclinic, $P 2_{1} / c$

130

8.7190 (3), 12.0891 (3), 24.6794 (7)

$90.806(3)$

2601.07 (13)

4

$\mathrm{Cu} K \alpha$

0.67

$0.41 \times 0.19 \times 0.04$

Rigaku Oxford Diffraction SuperNova, Dual,

$\mathrm{Cu}$ at zero, Atlas

Multi-scan (CrysAlis PRO; Rigaku OD, 2015)

$0.911,1.000$

$15892,5318,4425$

0.033

0.629
(1) $\cdot \mathrm{H}_{2} \mathrm{O}$

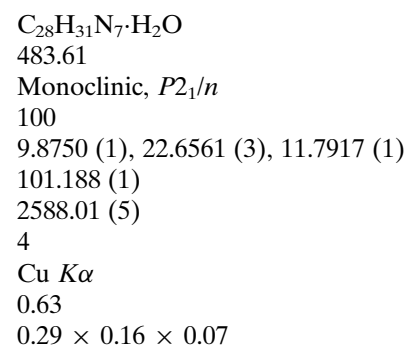

XtaLAB Synergy, Dualflex, HyPix

Multi-scan (CrysAlis PRO; Rigaku OD, 2015) $0.724,1.000$

$33621,5468,4622$

0.072

0.635

$0.050,0.144,1.08$
5468
345
$\mathrm{H}$ atoms treated by a mixture of independent
$\quad$ and constrained refinement
$0.51,-0.31$
Refinement

$R\left[F^{2}>2 \sigma\left(F^{2}\right)\right], w R\left(F^{2}\right), S$

No. of reflections

No. of parameters

H-atom treatment

$\Delta \rho_{\max }, \Delta \rho_{\min }\left(\mathrm{e} \AA^{-3}\right)$
$0.044,0.119,1.03$
5318
362
H atoms treated by a mixture of independent
$\quad$ and constrained refinement
$0.21,-0.24$

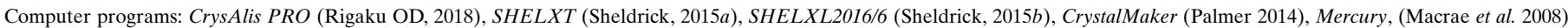
and publCIF (Westrip, 2010). 
molecule. The water molecule participates in two $\mathrm{O}-\mathrm{H} \cdots \mathrm{N}$ hydrogen bonds as donor and one $\mathrm{N}-\mathrm{H} \cdots \mathrm{O}$ hydrogen bond as acceptor to form a cluster of three molecules of (1) (Fig. 8 and Table 4). This cluster is then further hydrogen bonded via $\mathrm{N}-\mathrm{H} \cdots \mathrm{N}$ interactions between the remaining benzimidazolebased hydrogen-bond donors and acceptors (Fig. 9 and Table 4), to form two-dimensional hydrogen-bonded sheets lying in the (101) plane (Fig. 10).

\section{Database survey}

A search of the CSD (version 1.23; Groom et al., 2016) for structures related to (1) uncovered no hits.

\section{Synthesis and crystallization}

The synthesis of methylproamine (1) has been previously reported (Martin et al., 2004) but previous attempts to obtain crystals of the free ligand of suitable quality for X-ray analysis were not successful. In this study, crystals were serendipidously obtained during an attempt to obtain crystals of (1) complexed to $\beta$-cyclodextrin. Thus a solution of (1) $(6.8 \mathrm{mg})$ in $1 \mathrm{ml}$ of water saturated with $\beta$-cyclodextrin was left in a vapour diffusion tank with acetone allowed to diffuse into the solution. It is worth noting that (1) has very low solubility in water in the absence of $\beta$-cyclodextrin. After $12 \mathrm{~h}$, brown plates of (1) as its dihydrate developed, which were then harvested for X-ray analysis. The resulting solution when left to evaporate over a period of several months gave further needle-like crystals in a viscous matrix of $\beta$-cyclodextrin that were shown to be the monohydrate $(1) \cdot \mathrm{H}_{2} \mathrm{O}$.

\section{Refinement}

Crystal data, data collection and structure refinement details for (1) $\cdot 2 \mathrm{H}_{2} \mathrm{O}$ and (1) $\cdot \mathrm{H}_{2} \mathrm{O}$ are summarized in Table 5. In both structures, carbon-bound $\mathrm{H}$ atoms were placed in calculated positions and refined using a riding model, with methyl $\mathrm{C}-\mathrm{H}$ $=0.96 \AA$ and aromatic $\mathrm{C}-\mathrm{H}=0.93 \AA$ and $U_{\text {iso }}(\mathrm{H})=1.5 U_{\text {eq }}(\mathrm{C})$ for methyl and $1.2 U_{\mathrm{eq}}(\mathrm{C})$ for aromatic $\mathrm{C}-\mathrm{H}$. Hydrogen atoms attached to $\mathrm{N}$ and $\mathrm{O}$ were located in difference maps and allowed to refine with isotropic displacement parameters. In the structure of (1) $\cdot \mathrm{H}_{2} \mathrm{O}$ there are solvent-accessible voids of $154 \AA^{3}$ per unit cell; however, there was no significant difference electron density associated with these voids. The largest difference electron density of 0.5 e $\AA^{3}$ was associated with the piperazine group. Application of the SQUEEZE procedure (Spek, 2015) found eight electrons associated with the voids.

\section{Funding information}

Funding for this research was provided by: Sirtex Medical.

\section{References}

Groom, C. R., Bruno, I. J., Lightfoot, M. P. \& Ward, S. C. (2016). Acta Cryst. B72, 171-179.

Macrae, C. F., Bruno, I. J., Chisholm, J. A., Edgington, P. R., McCabe, P., Pidcock, E., Rodriguez-Monge, L., Taylor, R., van de Streek, J. \& Wood, P. A. (2008). J. Appl. Cryst. 41, 466-470.

Martin, R. F., Broadhurst, S., Reum, M. E., Squire, C. J., Clark, G. R., Lobachevsky, P. N., White, J. M., Clark, C., Sy, D., SpotheimMaurizot, M. \& Kelly, D. P. (2004). Cancer Res. 64, 1067-1070.

Palmer, D. C. (2014). CrystalMaker. CrystalMaker Software Ltd, Begbroke, England.

Pjura, P. E., Grzeskowiak, G. \& Dickerson, R. E. (1987). J. Mol. Biol. 197, 257-271.

Rigaku OD (2018). CrysAlis PRO. Rigaku Oxford Diffraction Ltd, Yarnton, England.

Sheldrick, G. M. (2015a). Acta Cryst. C71, 3-8.

Sheldrick, G. M. (2015b). Acta Cryst. A71, 3-8.

Spek, A. L. (2015). Acta Cryst. C71, 9-18.

Westrip, S. P. (2010). J. Appl. Cryst. 43, 920-925. 


\section{supporting information}

Acta Cryst. (2018). E74, 1903-1907 [https://doi.org/10.1107/S2056989018016791]

\section{Structures of the hydrate and dihydrate forms of the DNA-binding radioprotector methylproamine}

\section{Jonathan Michael White, Samuel Charles Brydon and Thomas Fellowes}

Computing details

Data collection: CrysAlis PRO (Rigaku OD, 2015) for 1_dihydrate; CrysAlis PRO (Rigaku OD, 2018) for 1_hydrate. Cell refinement: CrysAlis PRO (Rigaku OD, 2015) for 1_dihydrate; CrysAlis PRO (Rigaku OD, 2018) for 1_hydrate. Data reduction: CrysAlis PRO (Rigaku OD, 2015) for 1_dihydrate; CrysAlis PRO (Rigaku OD, 2018) for 1_hydrate. For both structures, program(s) used to solve structure: SHELXT (Sheldrick, 2015a); program(s) used to refine structure: SHELXL2016/6 (Sheldrick, 2015b). Molecular graphics: CrystalMaker (Palmer 2014) for 1_dihydrate; Mercury, (Macrae et al. 2008) for 1_hydrate. For both structures, software used to prepare material for publication: publCIF (Westrip, 2010).

N,N,3-Trimethyl-4-[6-(4-methylpiperazin-1-yl)-1H,3'H-[2,5'-bibenzo[d] imidazol]-2'-yl]aniline dihydrate

(1_dihydrate)

Crystal data

$\mathrm{C}_{28} \mathrm{H}_{31} \mathrm{~N}_{7} \cdot 2 \mathrm{H}_{2} \mathrm{O}$

$M_{r}=501.63$

Monoclinic, $P 2{ }_{1} / c$

$a=8.7190(3) \AA$

$b=12.0891$ (3) $\AA$

$c=24.6794(7) \AA$

$\beta=90.806(3)^{\circ}$

$V=2601.07(13) \AA^{3}$

$Z=4$

\section{Data collection}

Rigaku Oxford Diffraction SuperNova, Dual, $\mathrm{Cu}$ at zero, Atlas diffractometer

Radiation source: micro-focus sealed X-ray tube, SuperNova $(\mathrm{Cu})$ X-ray Source

Mirror monochromator

Detector resolution: 10.2273 pixels $\mathrm{mm}^{-1}$

$\omega$ scans

Absorption correction: multi-scan

(CrysAlisPro; Rigaku OD, 2015)

\section{Refinement}

Refinement on $F^{2}$

Least-squares matrix: full
$F(000)=1072$

$D_{\mathrm{x}}=1.281 \mathrm{Mg} \mathrm{m}^{-3}$

$\mathrm{Cu} K \alpha$ radiation, $\lambda=1.54184 \AA$

Cell parameters from 4889 reflections

$\theta=3.6-75.0^{\circ}$

$\mu=0.67 \mathrm{~mm}^{-1}$

$T=130 \mathrm{~K}$

PLATE, brown

$0.41 \times 0.19 \times 0.04 \mathrm{~mm}$

$T_{\min }=0.911, T_{\max }=1.000$

15892 measured reflections

5318 independent reflections

4425 reflections with $I>2 \sigma(I)$

$R_{\text {int }}=0.033$

$\theta_{\max }=75.8^{\circ}, \theta_{\min }=3.6^{\circ}$

$h=-10 \rightarrow 10$

$k=-15 \rightarrow 14$

$l=-16 \rightarrow 30$

$R\left[F^{2}>2 \sigma\left(F^{2}\right)\right]=0.044$

$w R\left(F^{2}\right)=0.119$ 
$S=1.03$

5318 reflections

362 parameters

0 restraints

Hydrogen site location: mixed
$\mathrm{H}$ atoms treated by a mixture of independent and constrained refinement

$w=1 /\left[\sigma^{2}\left(F_{\mathrm{o}}^{2}\right)+(0.0583 P)^{2}+0.7255 P\right]$

where $P=\left(F_{\mathrm{o}}{ }^{2}+2 F_{\mathrm{c}}{ }^{2}\right) / 3$

$(\Delta / \sigma)_{\max }=0.001$

$\Delta \rho_{\max }=0.21 \mathrm{e} \AA^{-3}$

$\Delta \rho_{\min }=-0.24$ e $\AA^{-3}$

Special details

Geometry. All esds (except the esd in the dihedral angle between two 1.s. planes) are estimated using the full covariance matrix. The cell esds are taken into account individually in the estimation of esds in distances, angles and torsion angles; correlations between esds in cell parameters are only used when they are defined by crystal symmetry. An approximate (isotropic) treatment of cell esds is used for estimating esds involving 1.s. planes.

Fractional atomic coordinates and isotropic or equivalent isotropic displacement parameters $\left(\hat{A}^{2}\right)$

\begin{tabular}{lllll}
\hline & $x$ & $y$ & $z$ & $U_{\text {iso }} / U_{\text {eq }}$ \\
\hline C1 & $0.69302(17)$ & $0.17764(11)$ & $0.65874(5)$ & $0.0243(3)$ \\
C2 & $0.79427(17)$ & $0.11850(12)$ & $0.69258(6)$ & $0.0258(3)$ \\
C3 & $0.81413(17)$ & $0.00556(12)$ & $0.68405(6)$ & $0.0277(3)$ \\
H3 & 0.881999 & -0.033944 & 0.707464 & $0.033^{*}$ \\
C4 & $0.73832(17)$ & $-0.05232(12)$ & $0.64241(6)$ & $0.0270(3)$ \\
C5 & $0.64357(18)$ & $0.00932(12)$ & $0.60713(6)$ & $0.0287(3)$ \\
H5 & 0.594461 & -0.026113 & 0.577227 & $0.034^{*}$ \\
C6 & $0.62141(17)$ & $0.12092(12)$ & $0.61563(6)$ & $0.0268(3)$ \\
H6 & 0.555661 & 0.160674 & 0.591572 & $0.032^{*}$ \\
C7 & $0.65276(17)$ & $0.29433(11)$ & $0.66734(5)$ & $0.0242(3)$ \\
C8 & $0.56121(17)$ & $0.46080(11)$ & $0.64655(5)$ & $0.0244(3)$ \\
C9 & $0.49737(18)$ & $0.55453(11)$ & $0.62294(5)$ & $0.0257(3)$ \\
H9 & 0.479766 & 0.559215 & 0.584935 & $0.031^{*}$ \\
C10 & $0.46019(17)$ & $0.64149(11)$ & $0.65739(6)$ & $0.0253(3)$ \\
C11 & $0.48861(18)$ & $0.63285(12)$ & $0.71361(6)$ & $0.0281(3)$ \\
H11 & 0.464455 & 0.693767 & 0.736265 & $0.034^{*}$ \\
C12 & $0.55047(19)$ & $0.53861(12)$ & $0.73678(5)$ & $0.0287(3)$ \\
H12 & 0.566304 & 0.533360 & 0.774861 & $0.034^{*}$ \\
C13 & $0.58903(17)$ & $0.45133(11)$ & $0.70256(5)$ & $0.0249(3)$ \\
C14 & $0.38673(17)$ & $0.74118(11)$ & $0.63489(5)$ & $0.0248(3)$ \\
C15 & $0.27354(17)$ & $0.90514(12)$ & $0.63245(6)$ & $0.0253(3)$ \\
C16 & $0.21044(18)$ & $1.00914(12)$ & $0.64186(6)$ & $0.0272(3)$ \\
H16 & 0.196434 & 1.035778 & 0.677671 & $0.033^{*}$ \\
C17 & $0.16846(17)$ & $1.07293(12)$ & $0.59683(6)$ & $0.0287(3)$ \\
C18 & $0.1847(2)$ & $1.02899(13)$ & $0.54424(6)$ & $0.0330(3)$ \\
H18 & 0.153195 & 1.072271 & 0.513946 & $0.040^{*}$ \\
C19 & $0.2453(2)$ & $0.92474(13)$ & $0.53543(6)$ & $0.0318(3)$ \\
H19 & 0.254261 & 0.896344 & 0.499724 & $0.038^{*}$ \\
C20 & $0.29259(18)$ & $0.86253(12)$ & $0.58001(6)$ & $0.0266(3)$ \\
C21 & $0.2269(2)$ & $1.25553(12)$ & $0.63172(7)$ & $0.0340(3)$ \\
H21A & 0.314059 & 1.266312 & 0.607004 & $0.041^{*}$ \\
H21B & 0.266620 & 1.219978 & 0.665212 & $0.041^{*}$ \\
& & & &
\end{tabular}




\begin{tabular}{|c|c|c|c|c|}
\hline $\mathrm{C} 22$ & $0.1582(2)$ & $1.36723(13)$ & $0.64567(7)$ & $0.0378(4)$ \\
\hline $\mathrm{H} 22 \mathrm{~A}$ & 0.075915 & 1.357214 & 0.672463 & $0.045^{*}$ \\
\hline $\mathrm{H} 22 \mathrm{~B}$ & 0.238348 & 1.415039 & 0.662181 & $0.045^{*}$ \\
\hline $\mathrm{C} 23$ & $-0.0209(2)$ & $1.34796(14)$ & $0.57307(9)$ & $0.0430(4)$ \\
\hline $\mathrm{H} 23 \mathrm{~A}$ & -0.065811 & 1.383431 & 0.540348 & $0.052 *$ \\
\hline $\mathrm{H} 23 \mathrm{~B}$ & -0.104284 & 1.336053 & 0.599265 & $0.052 *$ \\
\hline $\mathrm{C} 24$ & $0.0488(2)$ & $1.23730(13)$ & $0.55780(8)$ & $0.0390(4)$ \\
\hline $\mathrm{H} 24 \mathrm{~A}$ & -0.030972 & 1.189565 & 0.541029 & $0.047 *$ \\
\hline $\mathrm{H} 24 \mathrm{~B}$ & 0.130560 & 1.248921 & 0.530945 & $0.047 *$ \\
\hline $\mathrm{C} 25$ & 0.88799 (19) & $0.17115(13)$ & $0.73735(6)$ & $0.0322(3)$ \\
\hline $\mathrm{H} 25 \mathrm{~A}$ & 0.832606 & 0.165570 & 0.771519 & $0.048^{*}$ \\
\hline $\mathrm{H} 25 \mathrm{~B}$ & 0.905626 & 0.249222 & 0.728749 & $0.048 *$ \\
\hline $\mathrm{H} 25 \mathrm{C}$ & 0.986732 & 0.132916 & 0.740906 & $0.048^{*}$ \\
\hline $\mathrm{C} 26$ & $0.7615(2)$ & $-0.21319(13)$ & $0.58283(7)$ & $0.0370(4)$ \\
\hline $\mathrm{H} 26 \mathrm{~A}$ & 0.686373 & -0.176899 & 0.558870 & $0.056^{*}$ \\
\hline $\mathrm{H} 26 \mathrm{~B}$ & 0.739873 & -0.292682 & 0.584254 & $0.056^{*}$ \\
\hline $\mathrm{H} 26 \mathrm{C}$ & 0.864900 & -0.201421 & 0.568883 & $0.056^{*}$ \\
\hline $\mathrm{C} 27$ & $0.8446(2)$ & $-0.22581(13)$ & $0.67654(7)$ & $0.0398(4)$ \\
\hline $\mathrm{H} 27 \mathrm{~A}$ & 0.953129 & -0.208560 & 0.671010 & $0.060^{*}$ \\
\hline H27B & 0.828282 & -0.305536 & 0.672196 & $0.060^{*}$ \\
\hline $\mathrm{H} 27 \mathrm{C}$ & 0.815422 & -0.203391 & 0.713128 & $0.060^{*}$ \\
\hline $\mathrm{C} 28$ & $0.0293(2)$ & $1.52855(14)$ & $0.61022(9)$ & $0.0481(5)$ \\
\hline $\mathrm{H} 28 \mathrm{~A}$ & 0.109769 & 1.577017 & 0.624928 & $0.072^{*}$ \\
\hline $\mathrm{H} 28 \mathrm{~B}$ & -0.051069 & 1.519072 & 0.637206 & $0.072 *$ \\
\hline $\mathrm{H} 28 \mathrm{C}$ & -0.015043 & 1.561809 & 0.577347 & $0.072 *$ \\
\hline N1 & $0.75161(17)$ & $-0.16655(10)$ & $0.63700(5)$ & $0.0326(3)$ \\
\hline $\mathrm{N} 2$ & $0.60315(15)$ & $0.35974(10)$ & $0.62531(5)$ & $0.0247(3)$ \\
\hline N3 & $0.64741(15)$ & $0.34701(10)$ & $0.71470(5)$ & 0.0258 \\
\hline N4 & $0.33347(15)$ & $0.82541(9)$ & $0.66670(5)$ & 0.0252 \\
\hline N5 & $0.36288(15)$ & $0.75965(10)$ & $0.58267(5)$ & $0.0276(3)$ \\
\hline N6 & $0.11305(15)$ & $1.18284(10)$ & $0.60580(5)$ & $0.0304(3)$ \\
\hline N7 & $0.09523(16)$ & 1.42065 (11) & $0.59706(6)$ & $0.0340(3)$ \\
\hline $\mathrm{O} 1$ & $0.71569(15)$ & $0.55306(10)$ & $0.49523(5)$ & $0.0367(3)$ \\
\hline $\mathrm{O} 2$ & $0.43370(15)$ & $0.65458(9)$ & 0.48653 (4) & $0.0320(2)$ \\
\hline H1A & $0.685(3)$ & $0.481(2)$ & $0.4987(10)$ & $0.064(7)^{*}$ \\
\hline H1B & $0.768(4)$ & $0.559(3)$ & $0.4623(13)$ & $0.088(10)^{*}$ \\
\hline $\mathrm{H} 2$ & $0.5989(19)$ & $0.3436(14)$ & $0.5915(7)$ & $0.024(4)^{*}$ \\
\hline $\mathrm{H} 2 \mathrm{~A}$ & $0.413(3)$ & $0.691(2)$ & $0.5155(9)$ & $0.049(6)^{*}$ \\
\hline $\mathrm{H} 2 \mathrm{~B}$ & $0.532(3)$ & $0.631(2)$ & $0.4886(11)$ & $0.079(9)^{*}$ \\
\hline $\mathrm{H} 4 \mathrm{~A}$ & $0.339(2)$ & $0.8289(15)$ & $0.7019(8)$ & $0.029(4)^{*}$ \\
\hline
\end{tabular}

Atomic displacement parameters $\left(\AA^{2}\right)$

\begin{tabular}{lllllll}
\hline & $U^{11}$ & $U^{22}$ & $U^{33}$ & $U^{12}$ & $U^{13}$ & $U^{23}$ \\
\hline C1 & $0.0348(7)$ & $0.0171(6)$ & $0.0212(6)$ & $0.0013(5)$ & $0.0027(5)$ & $0.0007(5)$ \\
C2 & $0.0335(7)$ & $0.0217(7)$ & $0.0222(6)$ & $-0.0003(6)$ & $0.0013(5)$ & $0.0005(5)$ \\
C3 & $0.0346(7)$ & $0.0222(7)$ & $0.0262(7)$ & $0.0041(6)$ & $-0.0018(6)$ & $0.0027(5)$ \\
C4 & $0.0350(7)$ & $0.0191(7)$ & $0.0269(7)$ & $0.0020(5)$ & $0.0028(6)$ & $0.0002(5)$
\end{tabular}




\begin{tabular}{|c|c|c|c|c|c|c|}
\hline $\mathrm{C} 5$ & $0.0392(8)$ & $0.0214(7)$ & $0.0256(7)$ & $0.0006(6)$ & $-0.0029(6)$ & $-0.0029(5)$ \\
\hline C6 & $0.0367(7)$ & $0.0211(7)$ & $0.0226(6)$ & $0.0037(6)$ & $-0.0010(5)$ & $0.0005(5)$ \\
\hline $\mathrm{C} 7$ & $0.0345(7)$ & $0.0182(6)$ & $0.0199(6)$ & $0.0004(5)$ & $0.0013(5)$ & $0.0017(5)$ \\
\hline $\mathrm{C} 8$ & $0.0369(7)$ & $0.0177(6)$ & $0.0186(6)$ & $-0.0003(5)$ & $0.0027(5)$ & $-0.0012(5)$ \\
\hline C9 & $0.0418(8)$ & $0.0188(6)$ & $0.0163(6)$ & $0.0023(6)$ & $0.0001(5)$ & $0.0002(5)$ \\
\hline $\mathrm{C} 10$ & $0.0382(7)$ & $0.0169(6)$ & $0.0207(6)$ & $0.0011(5)$ & $-0.0001(5)$ & $-0.0002(5)$ \\
\hline C11 & $0.0459(8)$ & $0.0182(6)$ & $0.0201(7)$ & $0.0032(6)$ & $0.0001(6)$ & -0.0030 \\
\hline $\mathrm{C} 12$ & $0.0477(8)$ & $0.0227(7)$ & $0.0156(6)$ & $0.0038(6)$ & $0.0000(6)$ & -0.0002 \\
\hline $\mathrm{C} 13$ & $0.0390(7)$ & $0.0171(6)$ & $0.0186(6)$ & $0.0010(5)$ & $0.0003(5)$ & $0.0018(5)$ \\
\hline $\mathrm{C} 14$ & $0.0389(7)$ & $0.0174(6)$ & $0.0181(6)$ & $0.0006(5)$ & $0.0004(5)$ & $-0.0016(5)$ \\
\hline $\mathrm{C} 15$ & $0.0350(7)$ & $0.0190(6)$ & $0.0219(7)$ & $0.0001(5)$ & $-0.0005(5)$ & $0.0015(5)$ \\
\hline $\mathrm{C} 16$ & $0.0379(7)$ & $0.0194(6)$ & $0.0243(7)$ & $0.0014(6)$ & $0.0019(5)$ & -0.0011 \\
\hline $\mathrm{C} 17$ & $0.0333(7)$ & $0.0193(7)$ & $0.0335(8)$ & $0.0008(6)$ & $-0.0003(6)$ & $0.0014(6)$ \\
\hline $\mathrm{C} 18$ & $0.0475(9)$ & $0.0232(7)$ & $0.0282(7)$ & $0.0007(6)$ & $-0.0072(6)$ & $0.0053(6)$ \\
\hline C19 & $0.0495(9)$ & $0.0242(7)$ & $0.0216(7)$ & $0.0020(6)$ & $-0.0041(6)$ & $-0.0009(5)$ \\
\hline $\mathrm{C} 20$ & $0.0392(8)$ & $0.0189(6)$ & $0.0217(7)$ & $0.0002(6)$ & $-0.0011(6)$ & -0.0002 \\
\hline $\mathrm{C} 21$ & $0.0434(9)$ & $0.0213(7)$ & $0.0369(8)$ & $0.0041(6)$ & $-0.0054(7)$ & $-0.0022(6)$ \\
\hline $\mathrm{C} 22$ & $0.0536(10)$ & $0.0219(7)$ & $0.0381(9)$ & $0.0051(7)$ & $0.0041(7)$ & $-0.0010(6)$ \\
\hline $\mathrm{C} 23$ & $0.0373(8)$ & $0.0280(8)$ & $0.0634(12)$ & $0.0063(7)$ & $-0.0037(8)$ & $0.0076(8)$ \\
\hline $\mathrm{C} 24$ & $0.0429(9)$ & $0.0237(7)$ & $0.0498(10)$ & $0.0025(7)$ & $-0.0131(7)$ & $0.0034(7)$ \\
\hline $\mathrm{C} 25$ & $0.0402(8)$ & $0.0264(7)$ & $0.0297(8)$ & $0.0028(6)$ & $-0.0055(6)$ & $-0.0016(6)$ \\
\hline C26 & $0.0538(10)$ & $0.0215(7)$ & $0.0358(8)$ & $0.0046(7)$ & $0.0007(7)$ & $-0.0045(6)$ \\
\hline $\mathrm{C} 27$ & $0.0573(10)$ & $0.0217(7)$ & $0.0403(9)$ & $0.0090(7)$ & $-0.0062(8)$ & $0.0018(6)$ \\
\hline $\mathrm{C} 28$ & $0.0591(11)$ & $0.0256(8)$ & $0.0598(12)$ & $0.0146(8)$ & $0.0114(9)$ & $0.0020(8)$ \\
\hline N1 & $0.0474(8)$ & $0.0185(6)$ & $0.0318(7)$ & $0.0049(5)$ & $-0.0021(6)$ & $-0.0010(5)$ \\
\hline $\mathrm{N} 2$ & $0.0410(7)$ & $0.0173(5)$ & $0.0158(6)$ & $0.0035(5)$ & $0.0012(5)$ & -0.0006 \\
\hline N3 & $0.0420(7)$ & $0.0171(5)$ & $0.0184(5)$ & $0.0017(5)$ & $0.0010(5)$ & $0.0012(4)$ \\
\hline N4 & $0.0411(7)$ & $0.0170(5)$ & $0.0175(6)$ & $0.0026(5)$ & $0.0007(5)$ & -0.0003 \\
\hline N5 & $0.0446(7)$ & $0.0185(6)$ & $0.0198(6)$ & $0.0031(5)$ & $-0.0013(5)$ & -0.0006 \\
\hline N6 & $0.0355(6)$ & $0.0193(6)$ & $0.0362(7)$ & $0.0027(5)$ & $-0.0012(5)$ & $0.0027(5)$ \\
\hline N7 & $0.0397(7)$ & $0.0197(6)$ & $0.0429(8)$ & $0.0059(5)$ & $0.0069(6)$ & $0.0029(5)$ \\
\hline $\mathrm{O} 1$ & $0.0456(6)$ & $0.0286(6)$ & $0.0360(6)$ & $0.0002(5)$ & $0.0037(5)$ & -0.0011 \\
\hline $\mathrm{O} 2$ & $0.0499(7)$ & $0.0276(5)$ & $0.0186(5)$ & $0.0081(5)$ & $-0.0014(4)$ & -0.0036 \\
\hline
\end{tabular}

Geometric parameters (A, $\left.{ }^{o}\right)$

\begin{tabular}{llll}
\hline $\mathrm{C} 1-\mathrm{C} 2$ & $1.403(2)$ & $\mathrm{C} 19-\mathrm{H} 19$ & 0.9500 \\
$\mathrm{C} 1-\mathrm{C} 6$ & $1.405(2)$ & $\mathrm{C} 20-\mathrm{N} 5$ & $1.3877(19)$ \\
$\mathrm{C} 1-\mathrm{C} 7$ & $1.4698(18)$ & $\mathrm{C} 21-\mathrm{N} 6$ & $1.466(2)$ \\
$\mathrm{C} 2-\mathrm{C} 3$ & $1.393(2)$ & $\mathrm{C} 21-\mathrm{C} 22$ & 0.9900 \\
$\mathrm{C} 2-\mathrm{C} 25$ & $1.506(2)$ & $\mathrm{C} 21-\mathrm{H} 21 \mathrm{~A}$ & 0.9900 \\
$\mathrm{C} 3-\mathrm{C} 4$ & $1.401(2)$ & $\mathrm{C} 21-\mathrm{H} 21 \mathrm{~B}$ & $1.463(2)$ \\
$\mathrm{C} 3-\mathrm{H} 3$ & 0.9500 & $\mathrm{C} 22-\mathrm{N} 7$ & 0.9900 \\
$\mathrm{C} 4-\mathrm{N} 1$ & $1.3923(18)$ & $\mathrm{C} 22-\mathrm{H} 22 \mathrm{~A}$ & 0.9900 \\
$\mathrm{C} 4-\mathrm{C} 5$ & $1.406(2)$ & $\mathrm{C} 22-\mathrm{H} 22 \mathrm{~B}$ & $1.460(2)$ \\
$\mathrm{C} 5-\mathrm{C} 6$ & $1.379(2)$ & $\mathrm{C} 23-\mathrm{N} 7$ & $1.519(2)$ \\
$\mathrm{C} 5-\mathrm{H} 5$ & 0.9500 & $\mathrm{C} 23-\mathrm{C} 24$ & 0.9900 \\
$\mathrm{C} 6-\mathrm{H} 6$ & 0.9500 & $\mathrm{C} 23-\mathrm{H} 23 \mathrm{~A}$ &
\end{tabular}




\begin{tabular}{|c|c|c|c|}
\hline $\mathrm{C} 7-\mathrm{N} 2$ & $1.3695(17)$ & $\mathrm{C} 23-\mathrm{H} 23 \mathrm{~B}$ & 0.9900 \\
\hline $\mathrm{C} 7-\mathrm{N} 3$ & $1.3325(18)$ & $\mathrm{C} 24-\mathrm{N} 6$ & $1.460(2)$ \\
\hline $\mathrm{C} 8-\mathrm{N} 2$ & $1.3808(17)$ & $\mathrm{C} 24-\mathrm{H} 24 \mathrm{~A}$ & 0.9900 \\
\hline $\mathrm{C} 8-\mathrm{C} 9$ & $1.3871(19)$ & $\mathrm{C} 24-\mathrm{H} 24 \mathrm{~B}$ & 0.9900 \\
\hline $\mathrm{C} 8-\mathrm{C} 13$ & $1.4047(19)$ & $\mathrm{C} 25-\mathrm{H} 25 \mathrm{~A}$ & 0.9800 \\
\hline $\mathrm{C} 9-\mathrm{C} 10$ & $1.3931(19)$ & $\mathrm{C} 25-\mathrm{H} 25 \mathrm{~B}$ & 0.9800 \\
\hline C9- $\mathrm{H} 9$ & 0.9500 & $\mathrm{C} 25-\mathrm{H} 25 \mathrm{C}$ & 0.9800 \\
\hline $\mathrm{C} 10-\mathrm{C} 11$ & $1.410(2)$ & $\mathrm{C} 26-\mathrm{N} 1$ & $1.455(2)$ \\
\hline $\mathrm{C} 10-\mathrm{C} 14$ & $1.4701(19)$ & $\mathrm{C} 26-\mathrm{H} 26 \mathrm{~A}$ & 0.9800 \\
\hline $\mathrm{C} 11-\mathrm{C} 12$ & $1.381(2)$ & $\mathrm{C} 26-\mathrm{H} 26 \mathrm{~B}$ & 0.9800 \\
\hline C11-H11 & 0.9500 & $\mathrm{C} 26-\mathrm{H} 26 \mathrm{C}$ & 0.9800 \\
\hline $\mathrm{C} 12-\mathrm{C} 13$ & $1.3957(19)$ & $\mathrm{C} 27-\mathrm{N} 1$ & $1.449(2)$ \\
\hline $\mathrm{C} 12-\mathrm{H} 12$ & 0.9500 & $\mathrm{C} 27-\mathrm{H} 27 \mathrm{~A}$ & 0.9800 \\
\hline $\mathrm{C} 13-\mathrm{N} 3$ & $1.3910(18)$ & $\mathrm{C} 27-\mathrm{H} 27 \mathrm{~B}$ & 0.9800 \\
\hline $\mathrm{C} 14-\mathrm{N} 4$ & $1.3708(18)$ & $\mathrm{C} 27-\mathrm{H} 27 \mathrm{C}$ & 0.9800 \\
\hline $\mathrm{C} 14-\mathrm{N} 5$ & $1.3215(18)$ & $\mathrm{C} 28-\mathrm{N} 7$ & $1.464(2)$ \\
\hline $\mathrm{C} 15-\mathrm{N} 4$ & $1.3799(18)$ & $\mathrm{C} 28-\mathrm{H} 28 \mathrm{~A}$ & 0.9800 \\
\hline $\mathrm{C} 15-\mathrm{C} 16$ & $1.393(2)$ & $\mathrm{C} 28-\mathrm{H} 28 \mathrm{~B}$ & 0.9800 \\
\hline $\mathrm{C} 15-\mathrm{C} 20$ & $1.4051(19)$ & $\mathrm{C} 28-\mathrm{H} 28 \mathrm{C}$ & 0.9800 \\
\hline $\mathrm{C} 16-\mathrm{C} 17$ & $1.397(2)$ & $\mathrm{N} 2-\mathrm{H} 2$ & $0.856(18)$ \\
\hline $\mathrm{C} 16-\mathrm{H} 16$ & 0.9500 & $\mathrm{~N} 4-\mathrm{H} 4 \mathrm{~A}$ & $0.870(19)$ \\
\hline $\mathrm{C} 17-\mathrm{C} 18$ & $1.412(2)$ & $\mathrm{O} 1-\mathrm{H} 1 \mathrm{~A}$ & $0.91(3)$ \\
\hline $\mathrm{C} 17-\mathrm{N} 6$ & $1.4321(19)$ & $\mathrm{O} 1-\mathrm{H} 1 \mathrm{~B}$ & $0.94(3)$ \\
\hline $\mathrm{C} 18-\mathrm{C} 19$ & $1.385(2)$ & $\mathrm{O} 2-\mathrm{H} 2 \mathrm{~A}$ & $0.86(2)$ \\
\hline C18-H18 & 0.9500 & $\mathrm{O} 2-\mathrm{H} 2 \mathrm{~B}$ & $0.90(3)$ \\
\hline $\mathrm{C} 19-\mathrm{C} 20$ & $1.390(2)$ & & \\
\hline $\mathrm{C} 2-\mathrm{C} 1-\mathrm{C} 6$ & $118.14(12)$ & $\mathrm{C} 22-\mathrm{C} 21-\mathrm{H} 21 \mathrm{~B}$ & 109.3 \\
\hline $\mathrm{C} 2-\mathrm{C} 1-\mathrm{C} 7$ & $123.56(13)$ & $\mathrm{H} 21 \mathrm{~A}-\mathrm{C} 21-\mathrm{H} 21 \mathrm{~B}$ & 108.0 \\
\hline $\mathrm{C} 6-\mathrm{C} 1-\mathrm{C} 7$ & $118.25(13)$ & $\mathrm{N} 7-\mathrm{C} 22-\mathrm{C} 21$ & $110.56(14$ \\
\hline $\mathrm{C} 3-\mathrm{C} 2-\mathrm{C} 1$ & $119.22(13)$ & $\mathrm{N} 7-\mathrm{C} 22-\mathrm{H} 22 \mathrm{~A}$ & 109.5 \\
\hline $\mathrm{C} 3-\mathrm{C} 2-\mathrm{C} 25$ & $117.25(13)$ & $\mathrm{C} 21-\mathrm{C} 22-\mathrm{H} 22 \mathrm{~A}$ & 109.5 \\
\hline $\mathrm{C} 1-\mathrm{C} 2-\mathrm{C} 25$ & $123.50(13)$ & $\mathrm{N} 7-\mathrm{C} 22-\mathrm{H} 22 \mathrm{~B}$ & 109.5 \\
\hline $\mathrm{C} 2-\mathrm{C} 3-\mathrm{C} 4$ & $122.83(13)$ & $\mathrm{C} 21-\mathrm{C} 22-\mathrm{H} 22 \mathrm{~B}$ & 109.5 \\
\hline $\mathrm{C} 2-\mathrm{C} 3-\mathrm{H} 3$ & 118.6 & $\mathrm{H} 22 \mathrm{~A}-\mathrm{C} 22-\mathrm{H} 22 \mathrm{~B}$ & 108.1 \\
\hline $\mathrm{C} 4-\mathrm{C} 3-\mathrm{H} 3$ & 118.6 & $\mathrm{~N} 7-\mathrm{C} 23-\mathrm{C} 24$ & $110.66(14)$ \\
\hline $\mathrm{N} 1-\mathrm{C} 4-\mathrm{C} 3$ & $121.77(13)$ & $\mathrm{N} 7-\mathrm{C} 23-\mathrm{H} 23 \mathrm{~A}$ & 109.5 \\
\hline $\mathrm{N} 1-\mathrm{C} 4-\mathrm{C} 5$ & $121.03(13)$ & $\mathrm{C} 24-\mathrm{C} 23-\mathrm{H} 23 \mathrm{~A}$ & 109.5 \\
\hline $\mathrm{C} 3-\mathrm{C} 4-\mathrm{C} 5$ & $117.17(13)$ & $\mathrm{N} 7-\mathrm{C} 23-\mathrm{H} 23 \mathrm{~B}$ & 109.5 \\
\hline $\mathrm{C} 6-\mathrm{C} 5-\mathrm{C} 4$ & $120.44(13)$ & $\mathrm{C} 24-\mathrm{C} 23-\mathrm{H} 23 \mathrm{~B}$ & 109.5 \\
\hline $\mathrm{C} 6-\mathrm{C} 5-\mathrm{H} 5$ & 119.8 & $\mathrm{H} 23 \mathrm{~A}-\mathrm{C} 23-\mathrm{H} 23 \mathrm{~B}$ & 108.1 \\
\hline $\mathrm{C} 4-\mathrm{C} 5-\mathrm{H} 5$ & 119.8 & $\mathrm{~N} 6-\mathrm{C} 24-\mathrm{C} 23$ & $110.25(15)$ \\
\hline $\mathrm{C} 5-\mathrm{C} 6-\mathrm{C} 1$ & $122.07(13)$ & $\mathrm{N} 6-\mathrm{C} 24-\mathrm{H} 24 \mathrm{~A}$ & 109.6 \\
\hline $\mathrm{C} 5-\mathrm{C} 6-\mathrm{H} 6$ & 119.0 & $\mathrm{C} 23-\mathrm{C} 24-\mathrm{H} 24 \mathrm{~A}$ & 109.6 \\
\hline $\mathrm{C} 1-\mathrm{C} 6-\mathrm{H} 6$ & 119.0 & $\mathrm{~N} 6-\mathrm{C} 24-\mathrm{H} 24 \mathrm{~B}$ & 109.6 \\
\hline $\mathrm{N} 3-\mathrm{C} 7-\mathrm{N} 2$ & $111.95(12)$ & $\mathrm{C} 23-\mathrm{C} 24-\mathrm{H} 24 \mathrm{~B}$ & 109.6 \\
\hline $\mathrm{N} 3-\mathrm{C} 7-\mathrm{C} 1$ & $126.63(12)$ & $\mathrm{H} 24 \mathrm{~A}-\mathrm{C} 24-\mathrm{H} 24 \mathrm{~B}$ & 108.1 \\
\hline $\mathrm{N} 2-\mathrm{C} 7-\mathrm{C} 1$ & $121.22(12)$ & $\mathrm{C} 2-\mathrm{C} 25-\mathrm{H} 25 \mathrm{~A}$ & 109.5 \\
\hline
\end{tabular}




\begin{tabular}{|c|c|c|c|}
\hline $\mathrm{N} 2-\mathrm{C} 8-\mathrm{C} 9$ & $132.08(13)$ & $\mathrm{C} 2-\mathrm{C} 25-\mathrm{H} 25 \mathrm{~B}$ & 109.5 \\
\hline $\mathrm{N} 2-\mathrm{C} 8-\mathrm{C} 13$ & $104.98(12)$ & $\mathrm{H} 25 \mathrm{~A}-\mathrm{C} 25-\mathrm{H} 25 \mathrm{~B}$ & 109.5 \\
\hline $\mathrm{C} 9-\mathrm{C} 8-\mathrm{C} 13$ & $122.86(12)$ & $\mathrm{C} 2-\mathrm{C} 25-\mathrm{H} 25 \mathrm{C}$ & 109.5 \\
\hline $\mathrm{C} 8-\mathrm{C} 9-\mathrm{C} 10$ & $117.10(12)$ & $\mathrm{H} 25 \mathrm{~A}-\mathrm{C} 25-\mathrm{H} 25 \mathrm{C}$ & 109.5 \\
\hline $\mathrm{C} 8-\mathrm{C} 9-\mathrm{H} 9$ & 121.4 & $\mathrm{H} 25 \mathrm{~B}-\mathrm{C} 25-\mathrm{H} 25 \mathrm{C}$ & 109.5 \\
\hline $\mathrm{C} 10-\mathrm{C} 9-\mathrm{H} 9$ & 121.4 & $\mathrm{~N} 1-\mathrm{C} 26-\mathrm{H} 26 \mathrm{~A}$ & 109.5 \\
\hline $\mathrm{C} 9-\mathrm{C} 10-\mathrm{C} 11$ & $120.36(13)$ & $\mathrm{N} 1-\mathrm{C} 26-\mathrm{H} 26 \mathrm{~B}$ & 109.5 \\
\hline $\mathrm{C} 9-\mathrm{C} 10-\mathrm{C} 14$ & $119.47(12)$ & $\mathrm{H} 26 \mathrm{~A}-\mathrm{C} 26-\mathrm{H} 26 \mathrm{~B}$ & 109.5 \\
\hline $\mathrm{C} 11-\mathrm{C} 10-\mathrm{C} 14$ & $120.14(12)$ & $\mathrm{N} 1-\mathrm{C} 26-\mathrm{H} 26 \mathrm{C}$ & 109.5 \\
\hline $\mathrm{C} 12-\mathrm{C} 11-\mathrm{C} 10$ & $122.06(13)$ & $\mathrm{H} 26 \mathrm{~A}-\mathrm{C} 26-\mathrm{H} 26 \mathrm{C}$ & 109.5 \\
\hline $\mathrm{C} 12-\mathrm{C} 11-\mathrm{H} 11$ & 119.0 & $\mathrm{H} 26 \mathrm{~B}-\mathrm{C} 26-\mathrm{H} 26 \mathrm{C}$ & 109.5 \\
\hline $\mathrm{C} 10-\mathrm{C} 11-\mathrm{H} 11$ & 119.0 & $\mathrm{~N} 1-\mathrm{C} 27-\mathrm{H} 27 \mathrm{~A}$ & 109.5 \\
\hline $\mathrm{C} 11-\mathrm{C} 12-\mathrm{C} 13$ & $117.98(13)$ & $\mathrm{N} 1-\mathrm{C} 27-\mathrm{H} 27 \mathrm{~B}$ & 109.5 \\
\hline $\mathrm{C} 11-\mathrm{C} 12-\mathrm{H} 12$ & 121.0 & $\mathrm{H} 27 \mathrm{~A}-\mathrm{C} 27-\mathrm{H} 27 \mathrm{~B}$ & 109.5 \\
\hline $\mathrm{C} 13-\mathrm{C} 12-\mathrm{H} 12$ & 121.0 & $\mathrm{~N} 1-\mathrm{C} 27-\mathrm{H} 27 \mathrm{C}$ & 109.5 \\
\hline $\mathrm{N} 3-\mathrm{C} 13-\mathrm{C} 12$ & $130.21(13)$ & $\mathrm{H} 27 \mathrm{~A}-\mathrm{C} 27-\mathrm{H} 27 \mathrm{C}$ & 109.5 \\
\hline $\mathrm{N} 3-\mathrm{C} 13-\mathrm{C} 8$ & $110.09(12)$ & $\mathrm{H} 27 \mathrm{~B}-\mathrm{C} 27-\mathrm{H} 27 \mathrm{C}$ & 109.5 \\
\hline $\mathrm{C} 12-\mathrm{C} 13-\mathrm{C} 8$ & $119.61(13)$ & $\mathrm{N} 7-\mathrm{C} 28-\mathrm{H} 28 \mathrm{~A}$ & 109.5 \\
\hline $\mathrm{N} 5-\mathrm{C} 14-\mathrm{N} 4$ & $112.53(12)$ & $\mathrm{N} 7-\mathrm{C} 28-\mathrm{H} 28 \mathrm{~B}$ & 109.5 \\
\hline $\mathrm{N} 5-\mathrm{C} 14-\mathrm{C} 10$ & $124.66(13)$ & $\mathrm{H} 28 \mathrm{~A}-\mathrm{C} 28-\mathrm{H} 28 \mathrm{~B}$ & 109.5 \\
\hline $\mathrm{N} 4-\mathrm{C} 14-\mathrm{C} 10$ & $122.81(12)$ & N7-C28-H28C & 109.5 \\
\hline $\mathrm{N} 4-\mathrm{C} 15-\mathrm{C} 16$ & $132.48(13)$ & $\mathrm{H} 28 \mathrm{~A}-\mathrm{C} 28-\mathrm{H} 28 \mathrm{C}$ & 109.5 \\
\hline $\mathrm{N} 4-\mathrm{C} 15-\mathrm{C} 20$ & $105.04(12)$ & $\mathrm{H} 28 \mathrm{~B}-\mathrm{C} 28-\mathrm{H} 28 \mathrm{C}$ & 109.5 \\
\hline $\mathrm{C} 16-\mathrm{C} 15-\mathrm{C} 20$ & $122.44(13)$ & $\mathrm{C} 4-\mathrm{N} 1-\mathrm{C} 27$ & $118.18(13)$ \\
\hline $\mathrm{C} 15-\mathrm{C} 16-\mathrm{C} 17$ & $117.71(13)$ & $\mathrm{C} 4-\mathrm{N} 1-\mathrm{C} 26$ & $118.60(13)$ \\
\hline $\mathrm{C} 15-\mathrm{C} 16-\mathrm{H} 16$ & 121.1 & $\mathrm{C} 27-\mathrm{N} 1-\mathrm{C} 26$ & $112.78(13)$ \\
\hline $\mathrm{C} 17-\mathrm{C} 16-\mathrm{H} 16$ & 121.1 & $\mathrm{C} 7-\mathrm{N} 2-\mathrm{C} 8$ & $107.81(11)$ \\
\hline $\mathrm{C} 16-\mathrm{C} 17-\mathrm{C} 18$ & $119.68(13)$ & $\mathrm{C} 7-\mathrm{N} 2-\mathrm{H} 2$ & $127.9(12)$ \\
\hline $\mathrm{C} 16-\mathrm{C} 17-\mathrm{N} 6$ & $118.32(13)$ & $\mathrm{C} 8-\mathrm{N} 2-\mathrm{H} 2$ & $124.2(12)$ \\
\hline $\mathrm{C} 18-\mathrm{C} 17-\mathrm{N} 6$ & $121.99(13)$ & $\mathrm{C} 7-\mathrm{N} 3-\mathrm{C} 13$ & $105.17(11)$ \\
\hline $\mathrm{C} 19-\mathrm{C} 18-\mathrm{C} 17$ & $122.03(14)$ & $\mathrm{C} 14-\mathrm{N} 4-\mathrm{C} 15$ & $107.25(12)$ \\
\hline $\mathrm{C} 19-\mathrm{C} 18-\mathrm{H} 18$ & 119.0 & $\mathrm{C} 14-\mathrm{N} 4-\mathrm{H} 4 \mathrm{~A}$ & $126.4(12)$ \\
\hline $\mathrm{C} 17-\mathrm{C} 18-\mathrm{H} 18$ & 119.0 & $\mathrm{C} 15-\mathrm{N} 4-\mathrm{H} 4 \mathrm{~A}$ & $126.3(12)$ \\
\hline $\mathrm{C} 18-\mathrm{C} 19-\mathrm{C} 20$ & $118.53(14)$ & $\mathrm{C} 14-\mathrm{N} 5-\mathrm{C} 20$ & $105.13(12)$ \\
\hline $\mathrm{C} 18-\mathrm{C} 19-\mathrm{H} 19$ & 120.7 & $\mathrm{C} 17-\mathrm{N} 6-\mathrm{C} 24$ & $114.80(13)$ \\
\hline $\mathrm{C} 20-\mathrm{C} 19-\mathrm{H} 19$ & 120.7 & $\mathrm{C} 17-\mathrm{N} 6-\mathrm{C} 21$ & $113.32(12)$ \\
\hline $\mathrm{N} 5-\mathrm{C} 20-\mathrm{C} 19$ & $130.40(13)$ & $\mathrm{C} 24-\mathrm{N} 6-\mathrm{C} 21$ & $109.48(12)$ \\
\hline $\mathrm{N} 5-\mathrm{C} 20-\mathrm{C} 15$ & $110.05(12)$ & $\mathrm{C} 23-\mathrm{N} 7-\mathrm{C} 22$ & $108.50(13)$ \\
\hline $\mathrm{C} 19-\mathrm{C} 20-\mathrm{C} 15$ & $119.54(13)$ & $\mathrm{C} 23-\mathrm{N} 7-\mathrm{C} 28$ & $110.72(14)$ \\
\hline $\mathrm{N} 6-\mathrm{C} 21-\mathrm{C} 22$ & $111.44(14)$ & $\mathrm{C} 22-\mathrm{N} 7-\mathrm{C} 28$ & $110.83(14)$ \\
\hline $\mathrm{N} 6-\mathrm{C} 21-\mathrm{H} 21 \mathrm{~A}$ & 109.3 & $\mathrm{H} 1 \mathrm{~A}-\mathrm{O} 1-\mathrm{H} 1 \mathrm{~B}$ & $108(2)$ \\
\hline $\mathrm{C} 22-\mathrm{C} 21-\mathrm{H} 21 \mathrm{~A}$ & 109.3 & $\mathrm{H} 2 \mathrm{~A}-\mathrm{O} 2-\mathrm{H} 2 \mathrm{~B}$ & $109(2)$ \\
\hline $\mathrm{N} 6-\mathrm{C} 21-\mathrm{H} 21 \mathrm{~B}$ & 109.3 & & \\
\hline $\mathrm{C} 6-\mathrm{C} 1-\mathrm{C} 2-\mathrm{C} 3$ & $-3.2(2)$ & $\mathrm{C} 18-\mathrm{C} 19-\mathrm{C} 20-\mathrm{N} 5$ & $-176.53(16)$ \\
\hline $\mathrm{C} 7-\mathrm{C} 1-\mathrm{C} 2-\mathrm{C} 3$ & $174.06(13)$ & $\mathrm{C} 18-\mathrm{C} 19-\mathrm{C} 20-\mathrm{C} 15$ & $2.0(2)$ \\
\hline $\mathrm{C} 6-\mathrm{C} 1-\mathrm{C} 2-\mathrm{C} 25$ & $174.91(14)$ & $\mathrm{N} 4-\mathrm{C} 15-\mathrm{C} 20-\mathrm{N} 5$ & $-0.20(17)$ \\
\hline $\mathrm{C} 7-\mathrm{C} 1-\mathrm{C} 2-\mathrm{C} 25$ & $-7.8(2)$ & $\mathrm{C} 16-\mathrm{C} 15-\mathrm{C} 20-\mathrm{N} 5$ & $177.83(14)$ \\
\hline
\end{tabular}




\begin{tabular}{|c|c|c|c|}
\hline $\mathrm{C} 1-\mathrm{C} 2-\mathrm{C} 3-\mathrm{C} 4$ & $0.8(2)$ & $\mathrm{N} 4-\mathrm{C} 15-\mathrm{C} 20-\mathrm{C} 19$ & $-178.99(14)$ \\
\hline $\mathrm{C} 25-\mathrm{C} 2-\mathrm{C} 3-\mathrm{C} 4$ & $-177.47(14)$ & $\mathrm{C} 16-\mathrm{C} 15-\mathrm{C} 20-\mathrm{C} 19$ & $-1.0(2)$ \\
\hline $\mathrm{C} 2-\mathrm{C} 3-\mathrm{C} 4-\mathrm{N} 1$ & $-175.39(14)$ & $\mathrm{N} 6-\mathrm{C} 21-\mathrm{C} 22-\mathrm{N} 7$ & $-57.69(19)$ \\
\hline $\mathrm{C} 2-\mathrm{C} 3-\mathrm{C} 4-\mathrm{C} 5$ & $2.5(2)$ & $\mathrm{N} 7-\mathrm{C} 23-\mathrm{C} 24-\mathrm{N} 6$ & $60.2(2)$ \\
\hline $\mathrm{N} 1-\mathrm{C} 4-\mathrm{C} 5-\mathrm{C} 6$ & $174.59(14)$ & $\mathrm{C} 3-\mathrm{C} 4-\mathrm{N} 1-\mathrm{C} 27$ & $1.2(2)$ \\
\hline $\mathrm{C} 3-\mathrm{C} 4-\mathrm{C} 5-\mathrm{C} 6$ & $-3.3(2)$ & $\mathrm{C} 5-\mathrm{C} 4-\mathrm{N} 1-\mathrm{C} 27$ & $-176.68(15)$ \\
\hline $\mathrm{C} 4-\mathrm{C} 5-\mathrm{C} 6-\mathrm{C} 1$ & $0.9(2)$ & $\mathrm{C} 3-\mathrm{C} 4-\mathrm{N} 1-\mathrm{C} 26$ & $-141.25(15)$ \\
\hline $\mathrm{C} 2-\mathrm{C} 1-\mathrm{C} 6-\mathrm{C} 5$ & $2.4(2)$ & $\mathrm{C} 5-\mathrm{C} 4-\mathrm{N} 1-\mathrm{C} 26$ & $40.9(2)$ \\
\hline $\mathrm{C} 7-\mathrm{C} 1-\mathrm{C} 6-\mathrm{C} 5$ & $-175.00(14)$ & $\mathrm{N} 3-\mathrm{C} 7-\mathrm{N} 2-\mathrm{C} 8$ & $-0.72(17)$ \\
\hline $\mathrm{C} 2-\mathrm{C} 1-\mathrm{C} 7-\mathrm{N} 3$ & $-30.0(2)$ & $\mathrm{C} 1-\mathrm{C} 7-\mathrm{N} 2-\mathrm{C} 8$ & $174.58(13)$ \\
\hline $\mathrm{C} 6-\mathrm{C} 1-\mathrm{C} 7-\mathrm{N} 3$ & $147.26(15)$ & $\mathrm{C} 9-\mathrm{C} 8-\mathrm{N} 2-\mathrm{C} 7$ & $-176.60(16)$ \\
\hline $\mathrm{C} 2-\mathrm{C} 1-\mathrm{C} 7-\mathrm{N} 2$ & $155.44(14)$ & $\mathrm{C} 13-\mathrm{C} 8-\mathrm{N} 2-\mathrm{C} 7$ & $0.18(16)$ \\
\hline $\mathrm{C} 6-\mathrm{C} 1-\mathrm{C} 7-\mathrm{N} 2$ & $-27.3(2)$ & $\mathrm{N} 2-\mathrm{C} 7-\mathrm{N} 3-\mathrm{C} 13$ & $0.92(17)$ \\
\hline $\mathrm{N} 2-\mathrm{C} 8-\mathrm{C} 9-\mathrm{C} 10$ & $176.15(15)$ & $\mathrm{C} 1-\mathrm{C} 7-\mathrm{N} 3-\mathrm{C} 13$ & $-174.06(14)$ \\
\hline $\mathrm{C} 13-\mathrm{C} 8-\mathrm{C} 9-\mathrm{C} 10$ & $-0.1(2)$ & $\mathrm{C} 12-\mathrm{C} 13-\mathrm{N} 3-\mathrm{C} 7$ & $175.75(16)$ \\
\hline $\mathrm{C} 8-\mathrm{C} 9-\mathrm{C} 10-\mathrm{C} 11$ & $0.5(2)$ & $\mathrm{C} 8-\mathrm{C} 13-\mathrm{N} 3-\mathrm{C} 7$ & $-0.79(17)$ \\
\hline $\mathrm{C} 8-\mathrm{C} 9-\mathrm{C} 10-\mathrm{C} 14$ & $-177.65(13)$ & $\mathrm{N} 5-\mathrm{C} 14-\mathrm{N} 4-\mathrm{C} 15$ & $-1.25(18)$ \\
\hline $\mathrm{C} 9-\mathrm{C} 10-\mathrm{C} 11-\mathrm{C} 12$ & $-1.4(2)$ & $\mathrm{C} 10-\mathrm{C} 14-\mathrm{N} 4-\mathrm{C} 15$ & $179.45(13)$ \\
\hline $\mathrm{C} 14-\mathrm{C} 10-\mathrm{C} 11-\mathrm{C} 12$ & $176.79(15)$ & $\mathrm{C} 16-\mathrm{C} 15-\mathrm{N} 4-\mathrm{C} 14$ & $-176.92(16)$ \\
\hline $\mathrm{C} 10-\mathrm{C} 11-\mathrm{C} 12-\mathrm{C} 13$ & $1.7(2)$ & $\mathrm{C} 20-\mathrm{C} 15-\mathrm{N} 4-\mathrm{C} 14$ & $0.83(16)$ \\
\hline $\mathrm{C} 11-\mathrm{C} 12-\mathrm{C} 13-\mathrm{N} 3$ & $-177.60(15)$ & $\mathrm{N} 4-\mathrm{C} 14-\mathrm{N} 5-\mathrm{C} 20$ & $1.09(17)$ \\
\hline $\mathrm{C} 11-\mathrm{C} 12-\mathrm{C} 13-\mathrm{C} 8$ & $-1.3(2)$ & $\mathrm{C} 10-\mathrm{C} 14-\mathrm{N} 5-\mathrm{C} 20$ & $-179.62(14)$ \\
\hline $\mathrm{N} 2-\mathrm{C} 8-\mathrm{C} 13-\mathrm{N} 3$ & $0.38(17)$ & $\mathrm{C} 19-\mathrm{C} 20-\mathrm{N} 5-\mathrm{C} 14$ & $178.09(17)$ \\
\hline $\mathrm{C} 9-\mathrm{C} 8-\mathrm{C} 13-\mathrm{N} 3$ & $177.53(14)$ & $\mathrm{C} 15-\mathrm{C} 20-\mathrm{N} 5-\mathrm{C} 14$ & $-0.53(17)$ \\
\hline $\mathrm{N} 2-\mathrm{C} 8-\mathrm{C} 13-\mathrm{C} 12$ & $-176.59(14)$ & $\mathrm{C} 16-\mathrm{C} 17-\mathrm{N} 6-\mathrm{C} 24$ & $-170.61(14)$ \\
\hline $\mathrm{C} 9-\mathrm{C} 8-\mathrm{C} 13-\mathrm{C} 12$ & $0.6(2)$ & $\mathrm{C} 18-\mathrm{C} 17-\mathrm{N} 6-\mathrm{C} 24$ & $10.8(2)$ \\
\hline $\mathrm{C} 9-\mathrm{C} 10-\mathrm{C} 14-\mathrm{N} 5$ & $-5.7(2)$ & $\mathrm{C} 16-\mathrm{C} 17-\mathrm{N} 6-\mathrm{C} 21$ & $62.58(19)$ \\
\hline $\mathrm{C} 11-\mathrm{C} 10-\mathrm{C} 14-\mathrm{N} 5$ & $176.15(15)$ & $\mathrm{C} 18-\mathrm{C} 17-\mathrm{N} 6-\mathrm{C} 21$ & $-115.97(17)$ \\
\hline $\mathrm{C} 9-\mathrm{C} 10-\mathrm{C} 14-\mathrm{N} 4$ & $173.54(14)$ & $\mathrm{C} 23-\mathrm{C} 24-\mathrm{N} 6-\mathrm{C} 17$ & $174.17(14)$ \\
\hline $\mathrm{C} 11-\mathrm{C} 10-\mathrm{C} 14-\mathrm{N} 4$ & $-4.6(2)$ & $\mathrm{C} 23-\mathrm{C} 24-\mathrm{N} 6-\mathrm{C} 21$ & $-57.08(18)$ \\
\hline $\mathrm{N} 4-\mathrm{C} 15-\mathrm{C} 16-\mathrm{C} 17$ & $176.01(15)$ & $\mathrm{C} 22-\mathrm{C} 21-\mathrm{N} 6-\mathrm{C} 17$ & $-174.13(13)$ \\
\hline $\mathrm{C} 20-\mathrm{C} 15-\mathrm{C} 16-\mathrm{C} 17$ & $-1.4(2)$ & $\mathrm{C} 22-\mathrm{C} 21-\mathrm{N} 6-\mathrm{C} 24$ & $56.31(18)$ \\
\hline $\mathrm{C} 15-\mathrm{C} 16-\mathrm{C} 17-\mathrm{C} 18$ & $2.7(2)$ & $\mathrm{C} 24-\mathrm{C} 23-\mathrm{N} 7-\mathrm{C} 22$ & $-59.96(19)$ \\
\hline $\mathrm{C} 15-\mathrm{C} 16-\mathrm{C} 17-\mathrm{N} 6$ & $-175.89(13)$ & $\mathrm{C} 24-\mathrm{C} 23-\mathrm{N} 7-\mathrm{C} 28$ & $178.21(15)$ \\
\hline $\mathrm{C} 16-\mathrm{C} 17-\mathrm{C} 18-\mathrm{C} 19$ & $-1.7(2)$ & $\mathrm{C} 21-\mathrm{C} 22-\mathrm{N} 7-\mathrm{C} 23$ & $58.40(18)$ \\
\hline $\mathrm{N} 6-\mathrm{C} 17-\mathrm{C} 18-\mathrm{C} 19$ & $176.81(15)$ & $\mathrm{C} 21-\mathrm{C} 22-\mathrm{N} 7-\mathrm{C} 28$ & $-179.83(15)$ \\
\hline $\mathrm{C} 17-\mathrm{C} 18-\mathrm{C} 19-\mathrm{C} 20$ & $-0.7(3)$ & & \\
\hline
\end{tabular}

Hydrogen-bond geometry $\left(A,{ }^{\circ}\right)$

\begin{tabular}{lllll}
\hline$D-\mathrm{H} \cdots A$ & $D-\mathrm{H}$ & $\mathrm{H} \cdots A$ & $D \cdots A$ & $D-\mathrm{H} \cdots A$ \\
\hline $\mathrm{C} 23-\mathrm{H} 23 B \cdots \mathrm{N} 2^{\mathrm{i}}$ & 0.99 & 2.65 & $3.542(2)$ & 149 \\
$\mathrm{O} 1-\mathrm{H} 1 A \cdots \mathrm{O} 2^{\mathrm{ii}}$ & $0.91(3)$ & $1.98(3)$ & $2.8665(17)$ & $164(2)$ \\
$\mathrm{O} 1-\mathrm{H} 1 B^{\cdots} \cdots \mathrm{N} 7^{\mathrm{iii}}$ & $0.94(3)$ & $1.91(3)$ & $2.8482(18)$ & $170(3)$ \\
$\mathrm{N} 2-\mathrm{H} 2 \cdots \mathrm{O} 2^{\mathrm{ii}}$ & $0.856(18)$ & $1.944(18)$ & $2.7797(15)$ & $165.0(17)$ \\
$\mathrm{O} 2-\mathrm{H} 2 A \cdots \mathrm{N} 5$ & $0.86(2)$ & $1.91(2)$ & $2.7685(16)$ & $175(2)$
\end{tabular}




$\begin{array}{lllll}\mathrm{O} 2-\mathrm{H} 2 B \cdots \mathrm{O} 1 & 0.90(3) & 1.86(3) & 2.7537(18) & 168(3) \\ \mathrm{N} 4-\mathrm{H} 4 A \cdots \mathrm{N} 3^{\text {iv }} & 0.870(19) & 2.072(19) & 2.9411(16) & 176.7(17)\end{array}$

Symmetry codes: (i) $x-1, y+1, z$; (ii) $-x+1,-y+1,-z+1$; (iii) $-x+1,-y+2,-z+1$; (iv) $-x+1, y+1 / 2,-z+3 / 2$.

N,N,3-Trimethyl-4-[6-(4-methylpiperazin-1-yl)-1 H,3'H-[2,5'-bibenzo[d]imidazol]-2'-yl]aniline monohydrate (1_hydrate)

Crystal data

$\mathrm{C}_{28} \mathrm{H}_{31} \mathrm{~N}_{7} \cdot \mathrm{H}_{2} \mathrm{O}$

$F(000)=1032$

$M_{r}=483.61$

Monoclinic, $P 2_{1} / n$

$D_{\mathrm{x}}=1.241 \mathrm{Mg} \mathrm{m}^{-3}$

$a=9.8750(1) \AA$

$\mathrm{Cu} K \alpha$ radiation, $\lambda=1.54184 \AA$

$b=22.6561(3) \AA$

Cell parameters from 12480 reflections

$c=11.7917(1) \AA$

$\beta=101.188(1)^{\circ}$

$V=2588.01(5) \AA^{3}$

$\theta=4.3-77.4^{\circ}$

$Z=4$

$\mu=0.63 \mathrm{~mm}^{-1}$

$T=100 \mathrm{~K}$

ROD, brown

$0.29 \times 0.16 \times 0.07 \mathrm{~mm}$

\section{Data collection}

XtaLAB Synergy, Dualflex, HyPix diffractometer

Radiation source: micro-focus sealed X-ray tube, PhotonJet $(\mathrm{Cu}) \mathrm{X}$-ray Source

Mirror monochromator

$\omega$ scans

Absorption correction: multi-scan

(CrysAlisPro; Rigaku OD, 2015)

$T_{\min }=0.724, T_{\max }=1.000$

33621 measured reflections

5468 independent reflections

4622 reflections with $I>2 \sigma(I)$

$R_{\text {int }}=0.072$

$\theta_{\max }=78.2^{\circ}, \theta_{\min }=3.9^{\circ}$

$h=-12 \rightarrow 12$

$k=-13 \rightarrow 28$

$l=-14 \rightarrow 14$

\section{Refinement}

Refinement on $F^{2}$

Least-squares matrix: full

$R\left[F^{2}>2 \sigma\left(F^{2}\right)\right]=0.050$

$w R\left(F^{2}\right)=0.144$

$S=1.08$

5468 reflections

345 parameters

0 restraints
Hydrogen site location: mixed

$\mathrm{H}$ atoms treated by a mixture of independent and constrained refinement

$w=1 /\left[\sigma^{2}\left(F_{\mathrm{o}}^{2}\right)+(0.0744 P)^{2}+0.8256 P\right]$

where $P=\left(F_{\mathrm{o}}^{2}+2 F_{\mathrm{c}}^{2}\right) / 3$

$(\Delta / \sigma)_{\max }<0.001$

$\Delta \rho_{\max }=0.51$ e $\AA^{-3}$

$\Delta \rho_{\min }=-0.31 \mathrm{e} \AA^{-3}$

\section{Special details}

Geometry. All esds (except the esd in the dihedral angle between two 1.s. planes) are estimated using the full covariance matrix. The cell esds are taken into account individually in the estimation of esds in distances, angles and torsion angles; correlations between esds in cell parameters are only used when they are defined by crystal symmetry. An approximate (isotropic) treatment of cell esds is used for estimating esds involving l.s. planes.

Fractional atomic coordinates and isotropic or equivalent isotropic displacement parameters $\left(\AA^{2}\right)$

\begin{tabular}{lllll}
\hline & $x$ & $y$ & $z$ & $U_{\text {iso }} * U_{\text {eq }}$ \\
\hline C1 & $0.01456(15)$ & $0.36648(7)$ & $0.26721(12)$ & $0.0241(3)$ \\
C2 & $0.00764(15)$ & $0.41121(7)$ & $0.18284(13)$ & $0.0248(3)$ \\
C3 & $-0.11128(16)$ & $0.44501(7)$ & $0.15471(13)$ & $0.0271(3)$ \\
H3 & -0.114786 & 0.474253 & 0.098861 & $0.032 *$
\end{tabular}




\begin{tabular}{|c|c|c|c|c|}
\hline $\mathrm{C} 4$ & $-0.22698(16)$ & $0.43717(7)$ & $0.20671(13)$ & $0.0281(3)$ \\
\hline $\mathrm{C} 5$ & $-0.22147(16)$ & $0.39030(8)$ & $0.28626(14)$ & $0.0301(3)$ \\
\hline H5 & -0.297515 & 0.382171 & 0.319436 & $0.036^{*}$ \\
\hline C6 & $-0.10332(16)$ & $0.35642(7)$ & $0.31502(13)$ & $0.0273(3)$ \\
\hline H6 & -0.101718 & 0.325855 & 0.367918 & $0.033^{*}$ \\
\hline $\mathrm{C} 7$ & $0.14024(15)$ & $0.33245(7)$ & $0.31155(12)$ & $0.0229(3)$ \\
\hline $\mathrm{C} 8$ & $0.34140(15)$ & $0.29191(7)$ & $0.33857(12)$ & $0.0228(3)$ \\
\hline C9 & $0.47236(15)$ & $0.27141(7)$ & $0.33039(13)$ & $0.0241(3)$ \\
\hline H9 & 0.508496 & 0.277953 & 0.264282 & $0.029 *$ \\
\hline $\mathrm{C} 10$ & $0.54734(15)$ & $0.24077(7)$ & $0.42464(12)$ & $0.0234(3)$ \\
\hline $\mathrm{C} 11$ & $0.49178(15)$ & $0.23108(7)$ & $0.52482(12)$ & $0.0236(3)$ \\
\hline H11 & 0.544475 & 0.210978 & 0.586806 & $0.028^{*}$ \\
\hline $\mathrm{C} 12$ & $0.36138(15)$ & $0.25057(7)$ & $0.53357(12)$ & $0.0241(3)$ \\
\hline H12 & 0.324416 & 0.243352 & 0.599015 & $0.029 *$ \\
\hline C13 & $0.28837(15)$ & $0.28160(6)$ & $0.43910(13)$ & $0.0228(3)$ \\
\hline C14 & $0.68280(15)$ & $0.21645(6)$ & $0.41738(12)$ & $0.0223(3)$ \\
\hline C15 & $0.85786(15)$ & $0.18224(6)$ & $0.35357(13)$ & $0.0228(3)$ \\
\hline $\mathrm{C} 16$ & $0.95061(15)$ & $0.16217(7)$ & $0.28615(13)$ & $0.0252(3)$ \\
\hline H16 & 0.929305 & 0.164904 & 0.205961 & $0.030^{*}$ \\
\hline C17 & $1.07574(15)$ & 0.13799 (6) & $0.34208(13)$ & $0.0244(3)$ \\
\hline C18 & $1.10692(15)$ & $0.13588(7)$ & $0.46444(13)$ & $0.0263(3)$ \\
\hline H18 & 1.191352 & 0.120358 & 0.500963 & $0.032^{*}$ \\
\hline C19 & $1.01680(16)$ & $0.15595(7)$ & $0.53104(13)$ & $0.0266(3)$ \\
\hline H19 & 1.039331 & 0.154514 & 0.611317 & $0.032 *$ \\
\hline $\mathrm{C} 20$ & $0.89077(15)$ & $0.17849(6)$ & $0.47443(13)$ & $0.0231(3)$ \\
\hline $\mathrm{C} 21$ & $1.13723(19)$ & $0.11372(9)$ & $0.15611(15)$ & $0.0368(4)$ \\
\hline $\mathrm{H} 21 \mathrm{~A}$ & 1.145758 & 0.153897 & 0.129953 & $0.044^{*}$ \\
\hline H21B & 1.042476 & 0.101140 & 0.129598 & $0.044 *$ \\
\hline $\mathrm{C} 22$ & $1.2334(2)$ & $0.07369(9)$ & $0.10524(16)$ & 0.0392 (4) \\
\hline $\mathrm{H} 22 \mathrm{~A}$ & 1.219614 & 0.033188 & 0.127120 & $0.047 *$ \\
\hline $\mathrm{H} 22 \mathrm{~B}$ & 1.210701 & 0.076123 & 0.021566 & $0.047^{*}$ \\
\hline $\mathrm{C} 23$ & 1.41019 (19) & $0.08795(8)$ & $0.27129(15)$ & $0.0361(4)$ \\
\hline $\mathrm{H} 23 \mathrm{~A}$ & 1.505209 & 0.100072 & 0.298135 & $0.043^{*}$ \\
\hline $\mathrm{H} 23 \mathrm{~B}$ & 1.400472 & 0.047877 & 0.297564 & $0.043^{*}$ \\
\hline $\mathrm{C} 24$ & $1.31556(17)$ & $0.12828(8)$ & $0.32169(15)$ & $0.0329(4)$ \\
\hline $\mathrm{H} 24 \mathrm{~A}$ & 1.337798 & 0.126002 & 0.405395 & $0.039 *$ \\
\hline $\mathrm{H} 24 \mathrm{~B}$ & 1.329549 & 0.168699 & 0.299402 & $0.039 *$ \\
\hline $\mathrm{C} 25$ & $0.12476(17)$ & $0.42521(7)$ & $0.12195(15)$ & $0.0314(3)$ \\
\hline $\mathrm{H} 25 \mathrm{~A}$ & 0.134451 & 0.393832 & 0.069391 & $0.047 *$ \\
\hline $\mathrm{H} 25 \mathrm{~B}$ & 0.208865 & 0.429238 & 0.177969 & $0.047^{*}$ \\
\hline $\mathrm{H} 25 \mathrm{C}$ & 0.105481 & 0.461455 & 0.079748 & $0.047 *$ \\
\hline $\mathrm{C} 26$ & $-0.3390(2)$ & $0.52112(8)$ & $0.09748(18)$ & $0.0413(4)$ \\
\hline $\mathrm{H} 26 \mathrm{~A}$ & -0.265556 & 0.547916 & 0.127881 & $0.062 *$ \\
\hline H26B & -0.425557 & 0.541683 & 0.085972 & $0.062 *$ \\
\hline $\mathrm{H} 26 \mathrm{C}$ & -0.324627 & 0.505577 & 0.024996 & $0.062 *$ \\
\hline $\mathrm{C} 27$ & $-0.45043(19)$ & $0.47056(10)$ & $0.24402(18)$ & $0.0435(5)$ \\
\hline $\mathrm{H} 27 \mathrm{~A}$ & -0.493893 & 0.432503 & 0.234141 & $0.065^{*}$ \\
\hline H27B & -0.517460 & 0.500604 & 0.216914 & $0.065^{*}$ \\
\hline
\end{tabular}




$\begin{array}{lllll}\text { H27C } & -0.412649 & 0.476904 & 0.324428 & 0.065^{*} \\ \text { C28 } & 1.4660(2) & 0.04941(9) & 0.09566(18) & 0.0452(5) \\ \text { H28A } & 1.452215 & 0.009836 & 0.120255 & 0.068^{*} \\ \text { H28B } & 1.560756 & 0.060461 & 0.121696 & 0.068^{*} \\ \text { H28C } & 1.443296 & 0.051435 & 0.012819 & 0.068^{*} \\ \text { N1 } & -0.34070(14) & 0.47306(7) & 0.17847(13) & 0.0347(3) \\ \text { N2 } & 0.24614(13) & 0.32318(6) & 0.25861(11) & 0.0239(3) \\ \text { N3 } & 0.16059(13) & 0.30763(6) & 0.41935(11) & 0.0236(3) \\ \text { N4 } & 0.72636(13) & 0.20628(6) & 0.31942(11) & 0.0244(3) \\ \text { N5 } & 0.77794(13) & 0.20066(6) & 0.51316(11) & 0.0231(3) \\ \text { N6 } & 1.17078(13) & 0.11179(6) & 0.28103(11) & 0.0268(3) \\ \text { N7 } & 1.37733(15) & 0.08964(6) & 0.14459(12) & 0.0324(3) \\ \text { O1 } & 0.03124(13) & 0.30826(6) & 0.60438(10) & 0.0343(3) \\ \text { H1A } & -0.016(4) & 0.3420(17) & 0.618(3) & 0.091(11)^{*} \\ \text { H1B } & 0.097(3) & 0.3046(11) & 0.675(2) & 0.051(7)^{*} \\ \text { H3A } & 0.107(2) & 0.3088(9) & 0.476(2) & 0.039(6)^{*} \\ \text { H5A } & 0.765(2) & 0.1996(9) & 0.585(2) & 0.031(5)^{*} \\ \end{array}$

Atomic displacement parameters $\left(\AA^{2}\right)$

\begin{tabular}{|c|c|c|c|c|c|c|}
\hline & $U^{11}$ & $U^{22}$ & $U^{33}$ & $U^{12}$ & $U^{13}$ & $U^{23}$ \\
\hline $\mathrm{C} 1$ & $0.0212(7)$ & $0.0309(7)$ & $0.0192(7)$ & $0.0020(6)$ & $0.0016(5)$ & $-0.0043(5)$ \\
\hline $\mathrm{C} 2$ & $0.0229(7)$ & $0.0282(7)$ & $0.0219(7)$ & $0.0014(6)$ & $0.0013(5)$ & $-0.0035(5)$ \\
\hline $\mathrm{C} 3$ & $0.0256(7)$ & $0.0285(7)$ & $0.0254(7)$ & $0.0015(6)$ & $0.0006(6)$ & $-0.0019(6)$ \\
\hline $\mathrm{C} 4$ & $0.0221(7)$ & $0.0341(8)$ & $0.0262(7)$ & $0.0037(6)$ & $-0.0001(6)$ & $-0.0059(6)$ \\
\hline $\mathrm{C} 5$ & $0.0221(7)$ & $0.0427(9)$ & $0.0251(7)$ & $0.0027(6)$ & $0.0041(6)$ & $-0.0043(6)$ \\
\hline C6 & $0.0225(7)$ & $0.0362(8)$ & $0.0225(7)$ & $0.0016(6)$ & $0.0029(6)$ & $0.0003(6)$ \\
\hline $\mathrm{C} 7$ & $0.0207(7)$ & $0.0295(7)$ & $0.0183(6)$ & 0.0013 (5) & $0.0034(5)$ & $-0.0011(5)$ \\
\hline $\mathrm{C} 8$ & $0.0207(7)$ & $0.0302(7)$ & $0.0172(6)$ & $0.0014(5)$ & $0.0025(5)$ & $0.0001(5)$ \\
\hline $\mathrm{C} 9$ & $0.0215(7)$ & $0.0324(7)$ & 0.0188 (7) & $0.0011(6)$ & $0.0053(5)$ & $0.0016(6)$ \\
\hline $\mathrm{C} 10$ & $0.0205(7)$ & $0.0285(7)$ & $0.0212(7)$ & $-0.0004(5)$ & $0.0043(5)$ & $0.0002(5)$ \\
\hline C11 & $0.0226(7)$ & $0.0293(7)$ & $0.0182(7)$ & $0.0014(6)$ & $0.0018(5)$ & $0.0016(5)$ \\
\hline $\mathrm{C} 12$ & $0.0239(7)$ & $0.0307(7)$ & $0.0183(7)$ & $0.0001(6)$ & $0.0058(5)$ & $-0.0003(5)$ \\
\hline $\mathrm{C} 13$ & $0.0195(7)$ & $0.0292(7)$ & $0.0200(7)$ & $0.0011(5)$ & $0.0042(5)$ & -0.0009 (5) \\
\hline $\mathrm{C} 14$ & $0.0200(7)$ & $0.0274(7)$ & $0.0191(7)$ & $0.0005(5)$ & $0.0028(5)$ & $0.0011(5)$ \\
\hline $\mathrm{C} 15$ & $0.0201(7)$ & $0.0271(7)$ & $0.0212(7)$ & $-0.0002(5)$ & $0.0036(5)$ & $0.0020(5)$ \\
\hline $\mathrm{C} 16$ & $0.0242(7)$ & $0.0331(8)$ & $0.0187(7)$ & $0.0017(6)$ & 0.0054 (5) & 0.0017 (6) \\
\hline $\mathrm{C} 17$ & $0.0226(7)$ & $0.0272(7)$ & $0.0242(7)$ & $-0.0002(5)$ & $0.0068(6)$ & $0.0002(5)$ \\
\hline $\mathrm{C} 18$ & $0.0210(7)$ & $0.0325(8)$ & $0.0244(7)$ & $0.0025(6)$ & $0.0017(6)$ & $-0.0007(6)$ \\
\hline C19 & $0.0238(7)$ & $0.0348(8)$ & $0.0202(7)$ & $0.0022(6)$ & $0.0016(6)$ & $-0.0012(6)$ \\
\hline $\mathrm{C} 20$ & $0.0201(7)$ & $0.0278(7)$ & $0.0215(7)$ & $0.0003(5)$ & $0.0046(5)$ & -0.0005 \\
\hline $\mathrm{C} 21$ & $0.0329(9)$ & $0.0517(10)$ & $0.0268(8)$ & $0.0055(7)$ & $0.0081(7)$ & $0.0003(7)$ \\
\hline $\mathrm{C} 22$ & $0.0394(10)$ & $0.0496(10)$ & $0.0305(9)$ & $0.0007(8)$ & $0.0117(7)$ & $-0.0062(7)$ \\
\hline $\mathrm{C} 23$ & $0.0316(8)$ & $0.0454(10)$ & $0.0329(9)$ & $0.0093(7)$ & $0.0104(7)$ & $0.0043(7)$ \\
\hline $\mathrm{C} 24$ & $0.0257(8)$ & $0.0432(9)$ & $0.0309(8)$ & $0.0016(7)$ & $0.0082(6)$ & $-0.0037(7)$ \\
\hline $\mathrm{C} 25$ & $0.0295(8)$ & $0.0323(8)$ & $0.0335(8)$ & $0.0039(6)$ & $0.0088(6)$ & $0.0062(6)$ \\
\hline $\mathrm{C} 26$ & $0.0326(9)$ & $0.0393(9)$ & $0.0503(11)$ & $0.0119(7)$ & $0.0040(8)$ & $0.0043(8)$ \\
\hline $\mathrm{C} 27$ & $0.0297(9)$ & $0.0549(11)$ & $0.0465(11)$ & $0.0148(8)$ & $0.0086(8)$ & $-0.0015(9)$ \\
\hline
\end{tabular}




\begin{tabular}{lllllll} 
C28 & $0.0525(11)$ & $0.0468(10)$ & $0.0426(10)$ & $0.0168(9)$ & $0.0246(9)$ & $0.0038(8)$ \\
N1 & $0.0253(7)$ & $0.0422(8)$ & $0.0356(8)$ & $0.0100(6)$ & $0.0036(6)$ & $-0.0001(6)$ \\
N2 & $0.0209(6)$ & $0.0323(6)$ & $0.0181(6)$ & $0.0041(5)$ & $0.0031(4)$ & $0.0015(5)$ \\
N3 & $0.0203(6)$ & $0.0325(6)$ & $0.0186(6)$ & $0.0036(5)$ & $0.0055(5)$ & $-0.0002(5)$ \\
N4 & $0.0210(6)$ & $0.0328(6)$ & $0.0194(6)$ & $0.0029(5)$ & $0.0038(5)$ & $0.0026(5)$ \\
N5 & $0.0192(6)$ & $0.0324(6)$ & $0.0175(6)$ & $0.0023(5)$ & $0.0027(5)$ & $0.0002(5)$ \\
N6 & $0.0228(6)$ & $0.0338(7)$ & $0.0245(6)$ & $0.0012(5)$ & $0.0063(5)$ & $-0.0025(5)$ \\
N7 & $0.0331(7)$ & $0.0363(7)$ & $0.0312(7)$ & $0.0081(6)$ & $0.0152(6)$ & $0.0017(6)$ \\
O1 & $0.0325(6)$ & $0.0502(7)$ & $0.0213(6)$ & $0.0055(5)$ & $0.0077(5)$ & $0.0010(5)$ \\
\hline
\end{tabular}

Geometric parameters $\left(A,{ }^{\circ}\right)$

\begin{tabular}{|c|c|c|c|}
\hline $\mathrm{C} 1-\mathrm{C} 6$ & $1.407(2)$ & $\mathrm{C} 19-\mathrm{C} 20$ & $1.390(2)$ \\
\hline $\mathrm{C} 1-\mathrm{C} 2$ & $1.412(2)$ & $\mathrm{C} 19-\mathrm{H} 19$ & 0.9300 \\
\hline $\mathrm{C} 1-\mathrm{C} 7$ & $1.468(2)$ & $\mathrm{C} 20-\mathrm{N} 5$ & $1.3783(19)$ \\
\hline $\mathrm{C} 2-\mathrm{C} 3$ & $1.387(2)$ & $\mathrm{C} 21-\mathrm{N} 6$ & $1.446(2)$ \\
\hline $\mathrm{C} 2-\mathrm{C} 25$ & $1.509(2)$ & $\mathrm{C} 21-\mathrm{C} 22$ & $1.518(2)$ \\
\hline $\mathrm{C} 3-\mathrm{C} 4$ & 1.409 (2) & $\mathrm{C} 21-\mathrm{H} 21 \mathrm{~A}$ & 0.9700 \\
\hline $\mathrm{C} 3-\mathrm{H} 3$ & 0.9300 & $\mathrm{C} 21-\mathrm{H} 21 \mathrm{~B}$ & 0.9700 \\
\hline $\mathrm{C} 4-\mathrm{N} 1$ & $1.374(2)$ & $\mathrm{C} 22-\mathrm{N} 7$ & $1.453(2)$ \\
\hline $\mathrm{C} 4-\mathrm{C} 5$ & $1.411(2)$ & $\mathrm{C} 22-\mathrm{H} 22 \mathrm{~A}$ & 0.9700 \\
\hline $\mathrm{C} 5-\mathrm{C} 6$ & $1.383(2)$ & $\mathrm{C} 22-\mathrm{H} 22 \mathrm{~B}$ & 0.9700 \\
\hline $\mathrm{C} 5-\mathrm{H} 5$ & 0.9300 & $\mathrm{C} 23-\mathrm{N} 7$ & $1.467(2)$ \\
\hline C6-H6 & 0.9300 & $\mathrm{C} 23-\mathrm{C} 24$ & $1.509(2)$ \\
\hline $\mathrm{C} 7-\mathrm{N} 2$ & $1.3344(19)$ & $\mathrm{C} 23-\mathrm{H} 23 \mathrm{~A}$ & 0.9700 \\
\hline $\mathrm{C} 7-\mathrm{N} 3$ & 1.3687 (19) & $\mathrm{C} 23-\mathrm{H} 23 \mathrm{~B}$ & 0.9700 \\
\hline $\mathrm{C} 8-\mathrm{N} 2$ & $1.3899(19)$ & $\mathrm{C} 24-\mathrm{N} 6$ & $1.465(2)$ \\
\hline $\mathrm{C} 8-\mathrm{C} 9$ & $1.395(2)$ & $\mathrm{C} 24-\mathrm{H} 24 \mathrm{~A}$ & 0.9700 \\
\hline $\mathrm{C} 8-\mathrm{C} 13$ & $1.405(2)$ & $\mathrm{C} 24-\mathrm{H} 24 \mathrm{~B}$ & 0.9700 \\
\hline C9- $\mathrm{C} 10$ & $1.395(2)$ & $\mathrm{C} 25-\mathrm{H} 25 \mathrm{~A}$ & 0.9600 \\
\hline C9-H9 & 0.9300 & $\mathrm{C} 25-\mathrm{H} 25 \mathrm{~B}$ & 0.9600 \\
\hline $\mathrm{C} 10-\mathrm{C} 11$ & $1.413(2)$ & $\mathrm{C} 25-\mathrm{H} 25 \mathrm{C}$ & 0.9600 \\
\hline $\mathrm{C} 10-\mathrm{C} 14$ & $1.464(2)$ & $\mathrm{C} 26-\mathrm{N} 1$ & $1.451(2)$ \\
\hline $\mathrm{C} 11-\mathrm{C} 12$ & $1.384(2)$ & $\mathrm{C} 26-\mathrm{H} 26 \mathrm{~A}$ & 0.9600 \\
\hline $\mathrm{C} 11-\mathrm{H} 11$ & 0.9300 & $\mathrm{C} 26-\mathrm{H} 26 \mathrm{~B}$ & 0.9600 \\
\hline $\mathrm{C} 12-\mathrm{C} 13$ & $1.394(2)$ & $\mathrm{C} 26-\mathrm{H} 26 \mathrm{C}$ & 0.9600 \\
\hline $\mathrm{C} 12-\mathrm{H} 12$ & 0.9300 & $\mathrm{C} 27-\mathrm{N} 1$ & 1.449 (2) \\
\hline $\mathrm{C} 13-\mathrm{N} 3$ & $1.3712(19)$ & $\mathrm{C} 27-\mathrm{H} 27 \mathrm{~A}$ & 0.9600 \\
\hline $\mathrm{C} 14-\mathrm{N} 4$ & $1.3286(19)$ & $\mathrm{C} 27-\mathrm{H} 27 \mathrm{~B}$ & 0.9600 \\
\hline $\mathrm{C} 14-\mathrm{N} 5$ & $1.3691(19)$ & $\mathrm{C} 27-\mathrm{H} 27 \mathrm{C}$ & 0.9600 \\
\hline $\mathrm{C} 15-\mathrm{N} 4$ & $1.3936(19)$ & $\mathrm{C} 28-\mathrm{N} 7$ & $1.458(2)$ \\
\hline $\mathrm{C} 15-\mathrm{C} 16$ & $1.400(2)$ & $\mathrm{C} 28-\mathrm{H} 28 \mathrm{~A}$ & 0.9600 \\
\hline $\mathrm{C} 15-\mathrm{C} 20$ & $1.402(2)$ & $\mathrm{C} 28-\mathrm{H} 28 \mathrm{~B}$ & 0.9600 \\
\hline $\mathrm{C} 16-\mathrm{C} 17$ & $1.395(2)$ & $\mathrm{C} 28-\mathrm{H} 28 \mathrm{C}$ & 0.9600 \\
\hline $\mathrm{C} 16-\mathrm{H} 16$ & 0.9300 & $\mathrm{~N} 3-\mathrm{H} 3 \mathrm{~A}$ & $0.93(2)$ \\
\hline C17-C18 & $1.417(2)$ & $\mathrm{N} 5-\mathrm{H} 5 \mathrm{~A}$ & $0.89(2)$ \\
\hline C17-N6 & $1.4190(19)$ & $\mathrm{O} 1-\mathrm{H} 1 \mathrm{~A}$ & $0.93(4)$ \\
\hline $\mathrm{C} 18-\mathrm{C} 19$ & $1.374(2)$ & $\mathrm{O} 1-\mathrm{H} 1 \mathrm{~B}$ & $0.95(3)$ \\
\hline
\end{tabular}


C18-H18

C6- $\mathrm{C} 1-\mathrm{C} 2$

$\mathrm{C} 6-\mathrm{C} 1-\mathrm{C} 7$

$\mathrm{C} 2-\mathrm{C} 1-\mathrm{C} 7$

$\mathrm{C} 3-\mathrm{C} 2-\mathrm{C} 1$

$\mathrm{C} 3-\mathrm{C} 2-\mathrm{C} 25$

$\mathrm{C} 1-\mathrm{C} 2-\mathrm{C} 25$

$\mathrm{C} 2-\mathrm{C} 3-\mathrm{C} 4$

$\mathrm{C} 2-\mathrm{C} 3-\mathrm{H} 3$

$\mathrm{C} 4-\mathrm{C} 3-\mathrm{H} 3$

$\mathrm{N} 1-\mathrm{C} 4-\mathrm{C} 3$

$\mathrm{N} 1-\mathrm{C} 4-\mathrm{C} 5$

$\mathrm{C} 3-\mathrm{C} 4-\mathrm{C} 5$

$\mathrm{C} 6-\mathrm{C} 5-\mathrm{C} 4$

$\mathrm{C} 6-\mathrm{C} 5-\mathrm{H} 5$

$\mathrm{C} 4-\mathrm{C} 5-\mathrm{H} 5$

$\mathrm{C} 5-\mathrm{C} 6-\mathrm{C} 1$

$\mathrm{C} 5-\mathrm{C} 6-\mathrm{H} 6$

$\mathrm{C} 1-\mathrm{C} 6-\mathrm{H} 6$

$\mathrm{N} 2-\mathrm{C} 7-\mathrm{N} 3$

$\mathrm{N} 2-\mathrm{C} 7-\mathrm{C} 1$

N3-C7-C1

$\mathrm{N} 2-\mathrm{C} 8-\mathrm{C} 9$

$\mathrm{N} 2-\mathrm{C} 8-\mathrm{C} 13$

$\mathrm{C} 9-\mathrm{C} 8-\mathrm{C} 13$

$\mathrm{C} 10-\mathrm{C} 9-\mathrm{C} 8$

$\mathrm{C} 10-\mathrm{C} 9-\mathrm{H} 9$

$\mathrm{C} 8-\mathrm{C} 9-\mathrm{H} 9$

C9- $10-\mathrm{C} 11$

C9- $10-\mathrm{C} 14$

$\mathrm{C} 11-\mathrm{C} 10-\mathrm{C} 14$

$\mathrm{C} 12-\mathrm{C} 11-\mathrm{C} 10$

$\mathrm{C} 12-\mathrm{C} 11-\mathrm{H} 11$

$\mathrm{C} 10-\mathrm{C} 11-\mathrm{H} 11$

$\mathrm{C} 11-\mathrm{C} 12-\mathrm{C} 13$

$\mathrm{C} 11-\mathrm{C} 12-\mathrm{H} 12$

$\mathrm{C} 13-\mathrm{C} 12-\mathrm{H} 12$

N3- C13-C12

$\mathrm{N} 3-\mathrm{C} 13-\mathrm{C} 8$

$\mathrm{C} 12-\mathrm{C} 13-\mathrm{C} 8$

$\mathrm{N} 4-\mathrm{C} 14-\mathrm{N} 5$

$\mathrm{N} 4-\mathrm{C} 14-\mathrm{C} 10$

$\mathrm{N} 5-\mathrm{C} 14-\mathrm{C} 10$

N4- $15-\mathrm{C} 16$

N4-C15-C20

$\mathrm{C} 16-\mathrm{C} 15-\mathrm{C} 20$

C17-C16-C15
0.9300

$117.89(14)$

118.69 (14)

$123.34(13)$

$119.18(14)$

117.50 (14)

$123.31(13)$

$123.14(15)$

118.4

118.4

$120.91(15)$

$122.05(15)$

$117.02(14)$

$120.18(15)$

119.9

119.9

$122.42(15)$

118.8

118.8

$112.50(13)$

$126.94(13)$

$120.53(13)$

$129.60(13)$

$110.06(12)$

$120.34(13)$

117.80 (13)

121.1

121.1

$120.75(13)$

$119.36(13)$

119.85 (13)

122.05 (13)

119.0

119.0

116.47 (13)

121.8

121.8

131.98 (14)

105.46 (12)

122.57 (13)

112.69 (13)

$124.72(13)$

122.58 (13)

129.68 (13)

109.67 (13)

120.64 (13)

118.43 (14)

\begin{tabular}{|c|c|}
\hline $\mathrm{N} 6-\mathrm{C} 21-\mathrm{H} 21 \mathrm{~B}$ & 109.6 \\
\hline $\mathrm{C} 22-\mathrm{C} 21-\mathrm{H} 21 \mathrm{~B}$ & 109.6 \\
\hline $\mathrm{H} 21 \mathrm{~A}-\mathrm{C} 21-\mathrm{H} 21 \mathrm{~B}$ & 108.1 \\
\hline $\mathrm{N} 7-\mathrm{C} 22-\mathrm{C} 21$ & $111.93(15)$ \\
\hline $\mathrm{N} 7-\mathrm{C} 22-\mathrm{H} 22 \mathrm{~A}$ & 109.2 \\
\hline $\mathrm{C} 21-\mathrm{C} 22-\mathrm{H} 22 \mathrm{~A}$ & 109.2 \\
\hline $\mathrm{N} 7-\mathrm{C} 22-\mathrm{H} 22 \mathrm{~B}$ & 109.2 \\
\hline $\mathrm{C} 21-\mathrm{C} 22-\mathrm{H} 22 \mathrm{~B}$ & 109.2 \\
\hline $\mathrm{H} 22 \mathrm{~A}-\mathrm{C} 22-\mathrm{H} 22 \mathrm{~B}$ & 107.9 \\
\hline $\mathrm{N} 7-\mathrm{C} 23-\mathrm{C} 24$ & $110.73(14)$ \\
\hline $\mathrm{N} 7-\mathrm{C} 23-\mathrm{H} 23 \mathrm{~A}$ & 109.5 \\
\hline $\mathrm{C} 24-\mathrm{C} 23-\mathrm{H} 23 \mathrm{~A}$ & 109.5 \\
\hline $\mathrm{N} 7-\mathrm{C} 23-\mathrm{H} 23 \mathrm{~B}$ & 109.5 \\
\hline $\mathrm{C} 24-\mathrm{C} 23-\mathrm{H} 23 \mathrm{~B}$ & 109.5 \\
\hline $\mathrm{H} 23 \mathrm{~A}-\mathrm{C} 23-\mathrm{H} 23 \mathrm{~B}$ & 108.1 \\
\hline $\mathrm{N} 6-\mathrm{C} 24-\mathrm{C} 23$ & $110.86(14)$ \\
\hline $\mathrm{N} 6-\mathrm{C} 24-\mathrm{H} 24 \mathrm{~A}$ & 109.5 \\
\hline $\mathrm{C} 23-\mathrm{C} 24-\mathrm{H} 24 \mathrm{~A}$ & 109.5 \\
\hline $\mathrm{N} 6-\mathrm{C} 24-\mathrm{H} 24 \mathrm{~B}$ & 109.5 \\
\hline $\mathrm{C} 23-\mathrm{C} 24-\mathrm{H} 24 \mathrm{~B}$ & 109.5 \\
\hline $\mathrm{H} 24 \mathrm{~A}-\mathrm{C} 24-\mathrm{H} 24 \mathrm{~B}$ & 108.1 \\
\hline $\mathrm{C} 2-\mathrm{C} 25-\mathrm{H} 25 \mathrm{~A}$ & 109.5 \\
\hline $\mathrm{C} 2-\mathrm{C} 25-\mathrm{H} 25 \mathrm{~B}$ & 109.5 \\
\hline $\mathrm{H} 25 \mathrm{~A}-\mathrm{C} 25-\mathrm{H} 25 \mathrm{~B}$ & 109.5 \\
\hline $\mathrm{C} 2-\mathrm{C} 25-\mathrm{H} 25 \mathrm{C}$ & 109.5 \\
\hline $\mathrm{H} 25 \mathrm{~A}-\mathrm{C} 25-\mathrm{H} 25 \mathrm{C}$ & 109.5 \\
\hline $\mathrm{H} 25 \mathrm{~B}-\mathrm{C} 25-\mathrm{H} 25 \mathrm{C}$ & 109.5 \\
\hline $\mathrm{N} 1-\mathrm{C} 26-\mathrm{H} 26 \mathrm{~A}$ & 109.5 \\
\hline $\mathrm{N} 1-\mathrm{C} 26-\mathrm{H} 26 \mathrm{~B}$ & 109.5 \\
\hline $\mathrm{H} 26 \mathrm{~A}-\mathrm{C} 26-\mathrm{H} 26 \mathrm{~B}$ & 109.5 \\
\hline $\mathrm{N} 1-\mathrm{C} 26-\mathrm{H} 26 \mathrm{C}$ & 109.5 \\
\hline $\mathrm{H} 26 \mathrm{~A}-\mathrm{C} 26-\mathrm{H} 26 \mathrm{C}$ & 109.5 \\
\hline $\mathrm{H} 26 \mathrm{~B}-\mathrm{C} 26-\mathrm{H} 26 \mathrm{C}$ & 109.5 \\
\hline N1-C27-H27A & 109.5 \\
\hline $\mathrm{N} 1-\mathrm{C} 27-\mathrm{H} 27 \mathrm{~B}$ & 109.5 \\
\hline $\mathrm{H} 27 \mathrm{~A}-\mathrm{C} 27-\mathrm{H} 27 \mathrm{~B}$ & 109.5 \\
\hline $\mathrm{N} 1-\mathrm{C} 27-\mathrm{H} 27 \mathrm{C}$ & 109.5 \\
\hline $\mathrm{H} 27 \mathrm{~A}-\mathrm{C} 27-\mathrm{H} 27 \mathrm{C}$ & 109.5 \\
\hline $\mathrm{H} 27 \mathrm{~B}-\mathrm{C} 27-\mathrm{H} 27 \mathrm{C}$ & 109.5 \\
\hline $\mathrm{N} 7-\mathrm{C} 28-\mathrm{H} 28 \mathrm{~A}$ & 109.5 \\
\hline $\mathrm{N} 7-\mathrm{C} 28-\mathrm{H} 28 \mathrm{~B}$ & 109.5 \\
\hline $\mathrm{H} 28 \mathrm{~A}-\mathrm{C} 28-\mathrm{H} 28 \mathrm{~B}$ & 109.5 \\
\hline $\mathrm{N} 7-\mathrm{C} 28-\mathrm{H} 28 \mathrm{C}$ & 109.5 \\
\hline $\mathrm{H} 28 \mathrm{~A}-\mathrm{C} 28-\mathrm{H} 28 \mathrm{C}$ & 109.5 \\
\hline $\mathrm{H} 28 \mathrm{~B}-\mathrm{C} 28-\mathrm{H} 28 \mathrm{C}$ & 109.5 \\
\hline $\mathrm{C} 4-\mathrm{N} 1-\mathrm{C} 27$ & $120.55(15)$ \\
\hline
\end{tabular}




\begin{tabular}{|c|c|c|c|}
\hline $\mathrm{C} 17-\mathrm{C} 16-\mathrm{H} 16$ & 120.8 & $\mathrm{C} 4-\mathrm{N} 1-\mathrm{C} 26$ & $119.35(15)$ \\
\hline $\mathrm{C} 15-\mathrm{C} 16-\mathrm{H} 16$ & 120.8 & $\mathrm{C} 27-\mathrm{N} 1-\mathrm{C} 26$ & $119.08(14)$ \\
\hline $\mathrm{C} 16-\mathrm{C} 17-\mathrm{C} 18$ & $119.50(14)$ & $\mathrm{C} 7-\mathrm{N} 2-\mathrm{C} 8$ & $104.57(12)$ \\
\hline $\mathrm{C} 16-\mathrm{C} 17-\mathrm{N} 6$ & $122.47(13)$ & $\mathrm{C} 7-\mathrm{N} 3-\mathrm{C} 13$ & $107.39(12)$ \\
\hline $\mathrm{C} 18-\mathrm{C} 17-\mathrm{N} 6$ & $117.92(13)$ & $\mathrm{C} 7-\mathrm{N} 3-\mathrm{H} 3 \mathrm{~A}$ & $130.9(14)$ \\
\hline $\mathrm{C} 19-\mathrm{C} 18-\mathrm{C} 17$ & $122.29(14)$ & $\mathrm{C} 13-\mathrm{N} 3-\mathrm{H} 3 \mathrm{~A}$ & $121.5(14)$ \\
\hline $\mathrm{C} 19-\mathrm{C} 18-\mathrm{H} 18$ & 118.9 & $\mathrm{C} 14-\mathrm{N} 4-\mathrm{C} 15$ & $104.91(12)$ \\
\hline $\mathrm{C} 17-\mathrm{C} 18-\mathrm{H} 18$ & 118.9 & $\mathrm{C} 14-\mathrm{N} 5-\mathrm{C} 20$ & $106.94(12)$ \\
\hline $\mathrm{C} 18-\mathrm{C} 19-\mathrm{C} 20$ & $117.78(14)$ & $\mathrm{C} 14-\mathrm{N} 5-\mathrm{H} 5 \mathrm{~A}$ & $126.4(13)$ \\
\hline $\mathrm{C} 18-\mathrm{C} 19-\mathrm{H} 19$ & 121.1 & $\mathrm{C} 20-\mathrm{N} 5-\mathrm{H} 5 \mathrm{~A}$ & $126.0(13)$ \\
\hline $\mathrm{C} 20-\mathrm{C} 19-\mathrm{H} 19$ & 121.1 & $\mathrm{C} 17-\mathrm{N} 6-\mathrm{C} 21$ & $117.43(13)$ \\
\hline $\mathrm{N} 5-\mathrm{C} 20-\mathrm{C} 19$ & $132.90(14)$ & $\mathrm{C} 17-\mathrm{N} 6-\mathrm{C} 24$ & $115.25(13)$ \\
\hline $\mathrm{N} 5-\mathrm{C} 20-\mathrm{C} 15$ & $105.78(12)$ & $\mathrm{C} 21-\mathrm{N} 6-\mathrm{C} 24$ & $109.98(13)$ \\
\hline $\mathrm{C} 19-\mathrm{C} 20-\mathrm{C} 15$ & $121.32(14)$ & $\mathrm{C} 22-\mathrm{N} 7-\mathrm{C} 28$ & $110.09(16)$ \\
\hline $\mathrm{N} 6-\mathrm{C} 21-\mathrm{C} 22$ & $110.31(15)$ & $\mathrm{C} 22-\mathrm{N} 7-\mathrm{C} 23$ & $109.16(14)$ \\
\hline $\mathrm{N} 6-\mathrm{C} 21-\mathrm{H} 21 \mathrm{~A}$ & 109.6 & $\mathrm{C} 28-\mathrm{N} 7-\mathrm{C} 23$ & $110.83(14)$ \\
\hline $\mathrm{C} 22-\mathrm{C} 21-\mathrm{H} 21 \mathrm{~A}$ & 109.6 & $\mathrm{H} 1 \mathrm{~A}-\mathrm{O} 1-\mathrm{H} 1 \mathrm{~B}$ & $102(3)$ \\
\hline $\mathrm{C} 6-\mathrm{C} 1-\mathrm{C} 2-\mathrm{C} 3$ & $3.1(2)$ & $\mathrm{C} 18-\mathrm{C} 19-\mathrm{C} 20-\mathrm{N} 5$ & $178.45(16)$ \\
\hline $\mathrm{C} 7-\mathrm{C} 1-\mathrm{C} 2-\mathrm{C} 3$ & $-173.56(13)$ & $\mathrm{C} 18-\mathrm{C} 19-\mathrm{C} 20-\mathrm{C} 15$ & $-1.8(2)$ \\
\hline $\mathrm{C} 6-\mathrm{C} 1-\mathrm{C} 2-\mathrm{C} 25$ & $-177.96(14)$ & $\mathrm{N} 4-\mathrm{C} 15-\mathrm{C} 20-\mathrm{N} 5$ & $-0.14(16)$ \\
\hline $\mathrm{C} 7-\mathrm{C} 1-\mathrm{C} 2-\mathrm{C} 25$ & $5.4(2)$ & $\mathrm{C} 16-\mathrm{C} 15-\mathrm{C} 20-\mathrm{N} 5$ & $-178.90(14)$ \\
\hline $\mathrm{C} 1-\mathrm{C} 2-\mathrm{C} 3-\mathrm{C} 4$ & $0.2(2)$ & $\mathrm{N} 4-\mathrm{C} 15-\mathrm{C} 20-\mathrm{C} 19$ & $-179.98(14)$ \\
\hline $\mathrm{C} 25-\mathrm{C} 2-\mathrm{C} 3-\mathrm{C} 4$ & $-178.84(14)$ & $\mathrm{C} 16-\mathrm{C} 15-\mathrm{C} 20-\mathrm{C} 19$ & $1.3(2)$ \\
\hline $\mathrm{C} 2-\mathrm{C} 3-\mathrm{C} 4-\mathrm{N} 1$ & $177.91(14)$ & $\mathrm{N} 6-\mathrm{C} 21-\mathrm{C} 22-\mathrm{N} 7$ & $-57.8(2)$ \\
\hline $\mathrm{C} 2-\mathrm{C} 3-\mathrm{C} 4-\mathrm{C} 5$ & $-3.5(2)$ & $\mathrm{N} 7-\mathrm{C} 23-\mathrm{C} 24-\mathrm{N} 6$ & $58.11(19)$ \\
\hline $\mathrm{N} 1-\mathrm{C} 4-\mathrm{C} 5-\mathrm{C} 6$ & $-177.94(15)$ & $\mathrm{C} 3-\mathrm{C} 4-\mathrm{N} 1-\mathrm{C} 27$ & $-170.86(16)$ \\
\hline $\mathrm{C} 3-\mathrm{C} 4-\mathrm{C} 5-\mathrm{C} 6$ & $3.5(2)$ & $\mathrm{C} 5-\mathrm{C} 4-\mathrm{N} 1-\mathrm{C} 27$ & $10.6(2)$ \\
\hline $\mathrm{C} 4-\mathrm{C} 5-\mathrm{C} 6-\mathrm{C} 1$ & $-0.3(2)$ & $\mathrm{C} 3-\mathrm{C} 4-\mathrm{N} 1-\mathrm{C} 26$ & $-2.5(2)$ \\
\hline $\mathrm{C} 2-\mathrm{C} 1-\mathrm{C} 6-\mathrm{C} 5$ & $-3.1(2)$ & $\mathrm{C} 5-\mathrm{C} 4-\mathrm{N} 1-\mathrm{C} 26$ & $178.98(16)$ \\
\hline $\mathrm{C} 7-\mathrm{C} 1-\mathrm{C} 6-\mathrm{C} 5$ & $173.73(14)$ & $\mathrm{N} 3-\mathrm{C} 7-\mathrm{N} 2-\mathrm{C} 8$ & $-1.61(17)$ \\
\hline $\mathrm{C} 6-\mathrm{C} 1-\mathrm{C} 7-\mathrm{N} 2$ & $159.76(15)$ & $\mathrm{C} 1-\mathrm{C} 7-\mathrm{N} 2-\mathrm{C} 8$ & $176.41(14)$ \\
\hline $\mathrm{C} 2-\mathrm{C} 1-\mathrm{C} 7-\mathrm{N} 2$ & $-23.6(2)$ & $\mathrm{C} 9-\mathrm{C} 8-\mathrm{N} 2-\mathrm{C} 7$ & $-178.69(15)$ \\
\hline $\mathrm{C} 6-\mathrm{C} 1-\mathrm{C} 7-\mathrm{N} 3$ & $-22.4(2)$ & $\mathrm{C} 13-\mathrm{C} 8-\mathrm{N} 2-\mathrm{C} 7$ & $1.39(17)$ \\
\hline $\mathrm{C} 2-\mathrm{C} 1-\mathrm{C} 7-\mathrm{N} 3$ & $154.23(14)$ & $\mathrm{N} 2-\mathrm{C} 7-\mathrm{N} 3-\mathrm{C} 13$ & $1.24(17)$ \\
\hline $\mathrm{N} 2-\mathrm{C} 8-\mathrm{C} 9-\mathrm{C} 10$ & $-179.95(15)$ & $\mathrm{C} 1-\mathrm{C} 7-\mathrm{N} 3-\mathrm{C} 13$ & $-176.92(13)$ \\
\hline $\mathrm{C} 13-\mathrm{C} 8-\mathrm{C} 9-\mathrm{C} 10$ & $0.0(2)$ & $\mathrm{C} 12-\mathrm{C} 13-\mathrm{N} 3-\mathrm{C} 7$ & $179.70(16)$ \\
\hline $\mathrm{C} 8-\mathrm{C} 9-\mathrm{C} 10-\mathrm{C} 11$ & $-0.1(2)$ & $\mathrm{C} 8-\mathrm{C} 13-\mathrm{N} 3-\mathrm{C} 7$ & $-0.30(16)$ \\
\hline $\mathrm{C} 8-\mathrm{C} 9-\mathrm{C} 10-\mathrm{C} 14$ & $177.48(13)$ & $\mathrm{N} 5-\mathrm{C} 14-\mathrm{N} 4-\mathrm{C} 15$ & $0.15(17)$ \\
\hline $\mathrm{C} 9-\mathrm{C} 10-\mathrm{C} 11-\mathrm{C} 12$ & $0.9(2)$ & $\mathrm{C} 10-\mathrm{C} 14-\mathrm{N} 4-\mathrm{C} 15$ & $-178.70(14)$ \\
\hline $\mathrm{C} 14-\mathrm{C} 10-\mathrm{C} 11-\mathrm{C} 12$ & $-176.70(14)$ & $\mathrm{C} 16-\mathrm{C} 15-\mathrm{N} 4-\mathrm{C} 14$ & $178.62(16)$ \\
\hline $\mathrm{C} 10-\mathrm{C} 11-\mathrm{C} 12-\mathrm{C} 13$ & $-1.4(2)$ & $\mathrm{C} 20-\mathrm{C} 15-\mathrm{N} 4-\mathrm{C} 14$ & $0.00(16)$ \\
\hline $\mathrm{C} 11-\mathrm{C} 12-\mathrm{C} 13-\mathrm{N} 3$ & $-178.69(15)$ & $\mathrm{N} 4-\mathrm{C} 14-\mathrm{N} 5-\mathrm{C} 20$ & $-0.24(17)$ \\
\hline $\mathrm{C} 11-\mathrm{C} 12-\mathrm{C} 13-\mathrm{C} 8$ & $1.3(2)$ & $\mathrm{C} 10-\mathrm{C} 14-\mathrm{N} 5-\mathrm{C} 20$ & $178.64(13)$ \\
\hline $\mathrm{N} 2-\mathrm{C} 8-\mathrm{C} 13-\mathrm{N} 3$ & $-0.68(17)$ & $\mathrm{C} 19-\mathrm{C} 20-\mathrm{N} 5-\mathrm{C} 14$ & $-179.96(16)$ \\
\hline $\mathrm{C} 9-\mathrm{C} 8-\mathrm{C} 13-\mathrm{N} 3$ & $179.39(13)$ & $\mathrm{C} 15-\mathrm{C} 20-\mathrm{N} 5-\mathrm{C} 14$ & $0.22(16)$ \\
\hline $\mathrm{N} 2-\mathrm{C} 8-\mathrm{C} 13-\mathrm{C} 12$ & $179.32(14)$ & $\mathrm{C} 16-\mathrm{C} 17-\mathrm{N} 6-\mathrm{C} 21$ & $3.4(2)$ \\
\hline $\mathrm{C} 9-\mathrm{C} 8-\mathrm{C} 13-\mathrm{C} 12$ & $-0.6(2)$ & $\mathrm{C} 18-\mathrm{C} 17-\mathrm{N} 6-\mathrm{C} 21$ & $179.43(15)$ \\
\hline
\end{tabular}




\begin{tabular}{llll}
$\mathrm{C} 9-\mathrm{C} 10-\mathrm{C} 14-\mathrm{N} 4$ & $-19.8(2)$ & $\mathrm{C} 16-\mathrm{C} 17-\mathrm{N} 6-\mathrm{C} 24$ & $135.47(16)$ \\
$\mathrm{C} 11-\mathrm{C} 10-\mathrm{C} 14-\mathrm{N} 4$ & $157.83(15)$ & $\mathrm{C} 18-\mathrm{C} 17-\mathrm{N} 6-\mathrm{C} 24$ & $-48.47(19)$ \\
$\mathrm{C} 9-\mathrm{C} 10-\mathrm{C} 14-\mathrm{N} 5$ & $161.50(14)$ & $\mathrm{C} 22-\mathrm{C} 21-\mathrm{N} 6-\mathrm{C} 17$ & $-168.74(14)$ \\
$\mathrm{C} 11-\mathrm{C} 10-\mathrm{C} 14-\mathrm{N} 5$ & $-20.9(2)$ & $\mathrm{C} 22-\mathrm{C} 21-\mathrm{N} 6-\mathrm{C} 24$ & $56.83(19)$ \\
$\mathrm{N} 4-\mathrm{C} 15-\mathrm{C} 16-\mathrm{C} 17$ & $-177.97(14)$ & $\mathrm{C} 23-\mathrm{C} 24-\mathrm{N} 6-\mathrm{C} 17$ & $166.65(13)$ \\
$\mathrm{C} 20-\mathrm{C} 15-\mathrm{C} 16-\mathrm{C} 17$ & $0.5(2)$ & $\mathrm{C} 23-\mathrm{C} 24-\mathrm{N} 6-\mathrm{C} 21$ & $-57.84(18)$ \\
$\mathrm{C} 15-\mathrm{C} 16-\mathrm{C} 17-\mathrm{C} 18$ & $-1.7(2)$ & $\mathrm{C} 21-\mathrm{C} 22-\mathrm{N} 7-\mathrm{C} 28$ & $178.82(15)$ \\
$\mathrm{C} 15-\mathrm{C} 16-\mathrm{C} 17-\mathrm{N} 6$ & $174.29(14)$ & $\mathrm{C} 21-\mathrm{C} 22-\mathrm{N} 7-\mathrm{C} 23$ & $56.96(19)$ \\
$\mathrm{C} 16-\mathrm{C} 17-\mathrm{C} 18-\mathrm{C} 19$ & $1.2(2)$ & $\mathrm{C} 24-\mathrm{C} 23-\mathrm{N} 7-\mathrm{C} 22$ & $-56.93(19)$ \\
$\mathrm{N} 6-\mathrm{C} 17-\mathrm{C} 18-\mathrm{C} 19$ & $-174.97(15)$ & $\mathrm{C} 24-\mathrm{C} 23-\mathrm{N} 7-\mathrm{C} 28$ & $-178.34(16)$ \\
$\mathrm{C} 17-\mathrm{C} 18-\mathrm{C} 19-\mathrm{C} 20$ & $0.5(2)$ & & \\
\hline
\end{tabular}

Hydrogen-bond geometry $\left(\AA,{ }^{\circ}\right)$

\begin{tabular}{lllll}
\hline$D-\mathrm{H} \cdots A$ & $D-\mathrm{H}$ & $\mathrm{H} \cdots A$ & $D \cdots A$ & $D-\mathrm{H} \cdots A$ \\
\hline $\mathrm{O} 1-\mathrm{H} 1 A \cdots \mathrm{N} 7^{\mathrm{i}}$ & $0.93(4)$ & $1.93(4)$ & $2.858(2)$ & $177(3)$ \\
$\mathrm{O} 1-\mathrm{H} 1 B \cdots \mathrm{N} 4^{4 i}$ & $0.95(3)$ & $1.94(3)$ & $2.8905(18)$ & $177(2)$ \\
$\mathrm{N} 3-\mathrm{H} 3 A \cdots \mathrm{O} 1$ & $0.93(2)$ & $1.82(2)$ & $2.7338(17)$ & $170(2)$ \\
$\mathrm{N} 5-\mathrm{H} 5 A \cdots \mathrm{N} 2^{\mathrm{iii}}$ & $0.89(2)$ & $2.15(2)$ & $3.0199(18)$ & $167.2(18)$ \\
$\mathrm{O} 1-\mathrm{H} 1 A \cdots \mathrm{N} 7^{\mathrm{i}}$ & $0.93(4)$ & $1.93(4)$ & $2.858(2)$ & $177(3)$ \\
$\mathrm{O} 1-\mathrm{H} 1 B \cdots \mathrm{N} 4^{4 i}$ & $0.95(3)$ & $1.94(3)$ & $2.8905(18)$ & $177(2)$ \\
$\mathrm{N} 3-\mathrm{H} 3 A \cdots \mathrm{O} 1$ & $0.93(2)$ & $1.82(2)$ & $2.7338(17)$ & $170(2)$ \\
$\mathrm{N} 5-\mathrm{H} 5 A \cdots \mathrm{N} 2^{\mathrm{iii}}$ & $0.89(2)$ & $2.15(2)$ & $3.0199(18)$ & $167.2(18)$ \\
\hline
\end{tabular}

Symmetry codes: (i) $x-3 / 2,-y+1 / 2, z+1 / 2$; (ii) $x-1 / 2,-y+1 / 2, z+1 / 2$; (iii) $x+1 / 2,-y+1 / 2, z+1 / 2$. 